\title{
Magnetohydrodynamic Richtmyer-Meshkov instability under an arbitrarily oriented magnetic field
}

Cite as: Phys. Plasmas 27, 062101 (2020); https://doi.org/10.1063/1.5142042

Submitted: 10 December 2019 . Accepted: 08 May 2020. Published Online: 05 June 2020

Naijian Shen (D), Vincent Wheatley, D. I. Pullin, and Ravi Samtaney (D)

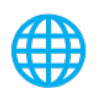




\title{
Magnetohydrodynamic Richtmyer-Meshkov instability under an arbitrarily oriented magnetic field
}

Cite as: Phys. Plasmas 27, 062101 (2020); doi: 10.1063/1.5142042

Submitted: 10 December 2019 • Accepted: 8 May 2020 .

Published Online: 5 June 2020

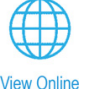

Naijian Shen, ${ }^{1, a)}$ iD Vincent Wheatley, ${ }^{2}$ D. I. Pullin, ${ }^{1}$ and Ravi Samtaney ${ }^{3}$

\begin{abstract}
AFFILIATIONS
${ }^{7}$ Graduate Aerospace Laboratories, California Institute of Technology, Pasadena, California 91125, USA

${ }^{2}$ School of Mechanical and Mining Engineering, University of Queensland, Queensland 4072, Australia

${ }^{3}$ Mechanical Engineering, Physical Science and Engineering Division, King Abdullah University of Science and Technology, Thuwal 23955-6900, Saudi Arabia
\end{abstract}

${ }^{a)}$ Author to whom correspondence should be addressed: nshen@caltech.edu

\begin{abstract}
The effect of an initially uniform magnetic field of arbitrary orientation on the Richtmyer-Meshkov instability in Hallmagnetohydrodynamics (MHD) and ideal MHD is considered. Attention is restricted to the case where the initial density interface has a single-mode sinusoidal perturbation in amplitude and is accelerated by a shock traveling perpendicular to the interface. An incompressible Hall-MHD model for this flow is developed by solving the relevant impulse-driven linearized initial value problem. The ideal MHD theory is naturally obtained by taking the limit of vanishing ion skin depth. It is shown that the out-of-plane magnetic field component normal to both the impulse and the interface perturbation does not affect the evolution of the flow. For all field orientations other than strictly out-ofplane, the growth of interface perturbations is suppressed. However, the suppression is most effective for near tangential fields but becomes less effective with increasing ion skin depth and Larmor radius. The modeled suppression mechanism is transport of vorticity along magnetic field lines via Alfvén fronts in ideal MHD, and via a dispersive wave system in Hall-MHD. Oscillation of the interface growth rate is caused by a continuous phase change of the induced velocities at the interface due to vorticity transport parallel to the perturbation direction in ideal MHD, while it can also result from interfacial vorticity production associated with the ion cyclotron effect in Hall-MHD with a finite Larmor radius. The limiting flow behavior of a large ion-skin-depth is explored. To assess the accuracy and appropriateness of the incompressible model, its ideal MHD predictions are compared to the results of the corresponding shock-driven nonlinear compressible simulations.
\end{abstract}

Published under license by AIP Publishing. https://doi.org/10.1063/1.5142042

\section{INTRODUCTION}

The perturbation growth of a shock-driven, impulsively accelerated, perturbed density interface, or contact discontinuity (CD) separating two fluids is known as the Richtmyer-Meshkov instability (RMI), due to the theoretical work of Ritchtmyer ${ }^{1}$ and the measurements of Meshkov. ${ }^{2}$ Typically characterized in a neutral gas, the RMI is pertinent in a variety of applications in astrophysics, ${ }^{3}$ combustion systems, ${ }^{4}$ chemical mixing, ${ }^{5}$ and more as surveyed in the review of Brouillette. ${ }^{6}$ More importantly, a richer literature is motivated by the development of inertial confinement fusion (ICF), a technology with the potential to demonstrate highly efficient carbon-free energy production. In ICF, a spherically converging shock is driven into a target capsule containing deuterium-tritium fuel, generating, in principle, a hot-spot at the center where fusion is initiated. The RMI, however, gives rise to mixing between the capsule material and the fuel within, compromising the chance of achieving energy break-even or production. ${ }^{8}$ Converging shock-driven RMI in both gases ${ }^{9-14}$ and solids ${ }^{15,16}$ has therefore received attention. Moreover, the high temperatures associated with ICF implosion inevitably cause rapid ionization of the involved materials, which then leads to the interaction between the conducting fluids and magnetic fields that are imposed or self-generated. ${ }^{17-20}$ Experiments by Hohenberger et $a .^{21}$ exploited this by immersing ICF targets in a near-uniform seed magnetic field before implosion, leading to enhanced compression and electron confinement. As a result, a significant increase in the ion temperature and neutron yield from the implosion was observed. In addition, two-dimensional radiation-hydrodynamics simulations by Perkins et al. ${ }^{22}$ show that uniform seed magnetic 
fields increase the robustness of ICF implosions to the outer perturbation amplitude.

Several theoretical descriptions have been proposed in order to model the coupled evolution of plasmas and magnetic fields. One framework is the single-fluid ideal magnetohydrodynamic (MHD) system, where it is demonstrated that the growth of the RMI is suppressed in the presence of an initially uniform magnetic field either normal $^{14,23-25}$ or tangential ${ }^{26,27}$ to the interface. The cylindrically and spherically converging MHD RMI is also suppressed under a range of seed magnetic field configurations, as investigated numerically by Mostert et al. ${ }^{28,29}$ In the cylindrical geometry, it is shown that while the suppression occurs along the entire density interface, its extent and the behavior of the interface are highly dependent on the local orientation of the magnetic field. Away from the ideal MHD region, where the ion cyclotron effect is significant, Shen et al. ${ }^{30}$ employed the incompressible Hall-MHD model to examine the impulse-driven RMI for the case where the initial magnetic field is normal to the interface. The suppression of the RMI is again established, although the vorticity transport mechanism responsible for such suppression differs markedly from that of the ideal MHD system. Using a more general twofluid plasma model, Bond et al. ${ }^{20}$ investigated computationally the planar shock-driven RMI and observed self-generated magnetic fields that are not initially imposed. In order of decreasing complexity, Shen et $a .^{31}$ showed that the two-fluid plasma equations, the Hall-MHD and ideal MHD models are connected via a series of limiting processes with respect to the appropriately scaled parameters including the speed-of-light, the ion skin depth, and the ion-to-electron mass ratio.

Extending the recent work of Shen et al. ${ }^{30}$ on a normal field, the present study utilizes the Hall-MHD equations to consider the effect of a uniform initial magnetic field of completely arbitrary orientation, on the RMI flow resulting from impulsively accelerating a density interface with a single-mode sinusoidal perturbation in amplitude. The linearized, incompressible, impulse-driven initial value problem, which admits an analytical solution in various limits, is considered to capture the leading-order features of the corresponding compressible shock-driven RM flow. The flow structure of the resulting analytical solutions is explored, with particular attention given to the dynamics of vorticity and the consequent evolution of the induced velocities at the interface. The ideal MHD theory of arbitrary field angle is naturally contained in this formulation as the limiting case of vanishing Larmor radius $d_{L}$, or equivalently, ion skin depth $d_{S}$. The adoption of the HallMHD model however enables access to the plasma region where $d_{L}, d_{S}>0$. Comparisons between the ideal MHD and Hall-MHD models are made throughout. The accuracy and validity of the incompressible formulation is assessed by a nonlinear simulation of the shock-driven compressible RM flow in the ideal MHD limit.

The rest of this paper is structured as follows: Sec. II first introduces the impulsive-driven initial value problem, governed by the incompressible Hall-MHD equations. These equations, together with the corresponding Rankine-Hugoniot conditions for a contact discontinuity are linearized around the unperturbed base-flow. The general flow field solution is then obtained in Sec. III for all field angles. The resulting interface behavior, as well as vorticity transport, is examined. Section IV derives limiting solutions for extreme values of a range of parameters, including the ion skin depth, the strength, and the direction of the initial magnetic field. The ideal MHD limit is discussed in detail. Illustrative results obtained from the linear theory and nonlinear simulation in terms of the interface perturbation growth, flow velocity, and vorticity profiles are given in Sec. V, before the conclusions are drawn in Sec. VI.

\section{INCOMPRESSIBLE HALL-MHD MODEL \\ A. Governing equations}

The initial condition for the impulse-driven RM flow under consideration is illustrated in Fig. 1(a). Cartesian coordinates are assigned so that the unperturbed interface lies in the $x, y$-plane, separating two fluids of densities $\rho_{1}(z<0)$ and $\rho_{2}(z>0)$. The single-mode perturbation of wavelength $\Lambda$ and amplitude $\eta_{0}$ varies its magnitude along the $x$-direction. The two-dimensional contact discontinuity (CD), defined by the interface, is subject to an impulsive acceleration given by $V_{0} \delta(t)$, where $V_{0}$ matches the imparted interface velocity from a shock-CD impact had it been traversed by an incident normal shock of Mach number $M$ in the positive $z$-direction given by the unit vector $\hat{z}$, and $\delta(t)$ is the Dirac delta function of time $t$ with the unit of frequency. In the non-inertial reference frame moving with the interface, a body force of the form $\rho V_{0} \delta(t) \hat{\boldsymbol{z}}$ is required for each fluid of density $\rho \in\left\{\rho_{1}, \rho_{2}\right\}$. The shock-wave-density-interface interaction can be analyzed by the solution to a suitable Riemann-type problem. A uniform initial magnetic field $\boldsymbol{B}_{0}$ of arbitrary orientation is imposed.

The non-dimensional variables are obtained by choosing the following reference scales: the perturbation wavelength $\Lambda$ for spatial coordinates, the post-shock interface speed $V_{0}$ for velocities, the applied field strength $B_{0}$ for magnetic fields, the elementary charge $e$ for particle charges, the ion mass $m_{i}$ for particle masses, and the left region fluid density $\rho_{1}$ for mass densities. These also lead to the derived reference quantities including the timescale $\Lambda / V_{0}$, the particle number density scale $\rho_{1} / m_{i}$, the pressure scale $\rho_{1} V_{0}^{2}$, the electric field scale $V_{0} B$, and the current density scale $e \rho_{1} V_{0} / m_{i}$. As a result, the dimensionless Hall-MHD equations that govern the evolution of the initial accelerative impulse for incompressible ions and electrons $\left(\mathrm{IIIE}^{30}\right)$ in the convenient non-inertial frame moving with the interface are given by

$$
\begin{gathered}
\nabla \cdot \boldsymbol{u}=0, \quad \nabla \cdot\left(\frac{\boldsymbol{j}}{\rho}\right)=0, \\
\rho\left(\frac{\partial \boldsymbol{u}}{\partial t}+\boldsymbol{u} \cdot \nabla \boldsymbol{u}\right)=-\nabla p+\frac{1}{d_{L}} \boldsymbol{j} \times \boldsymbol{B}+\boldsymbol{f}-\rho \delta(t) \hat{\boldsymbol{z}}, \\
\frac{\partial \boldsymbol{B}}{\partial t}+\nabla \times \boldsymbol{E}=\mathbf{0}, \\
\boldsymbol{E}+\boldsymbol{u} \times \boldsymbol{B}=\frac{\boldsymbol{j} \times \boldsymbol{B}}{\rho}-\frac{d_{L}}{\rho} \nabla p_{e}, \\
\nabla \times \boldsymbol{B}=\frac{\beta}{2 d_{L}} \boldsymbol{j}, \\
\nabla \cdot \boldsymbol{B}=0,
\end{gathered}
$$

where $\boldsymbol{u}=(u, v, w)$ is the flow velocity, $\rho$ is the mass density, $\boldsymbol{j}$ is the electric current density, $p$ is the total ion and electron pressure, $\boldsymbol{E}$ is the electric field, and $\boldsymbol{B}$ is the magnetic field. In the momentum equation (1b), the impulsive forcing that accelerates the fluids is given by

$$
\boldsymbol{f}=\left[\rho_{1}+H(z)\left(\rho_{2}-\rho_{1}\right)\right] \delta(t) \hat{\boldsymbol{z}} .
$$


(a)

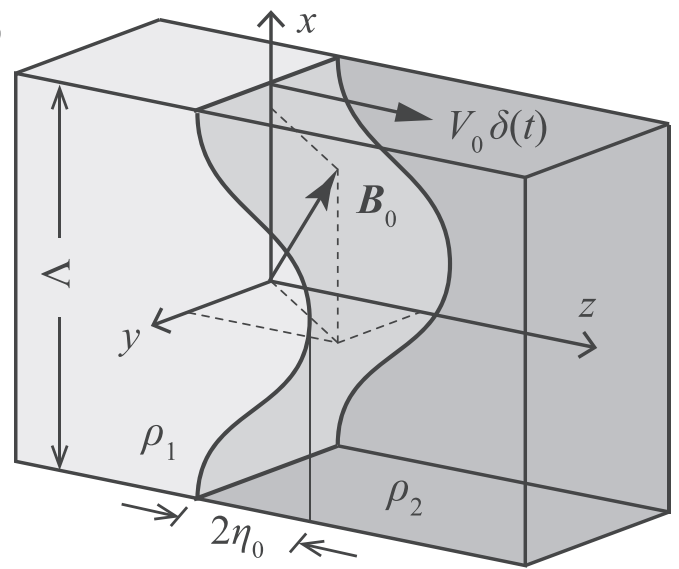

(b)

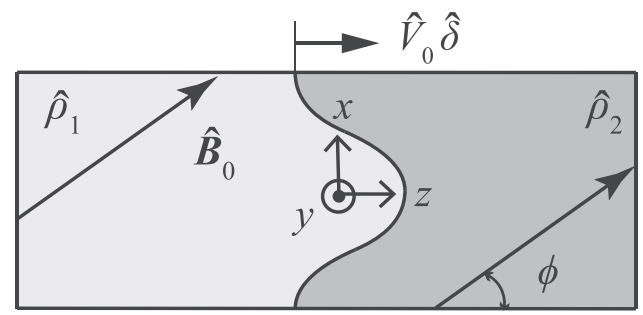

(c)

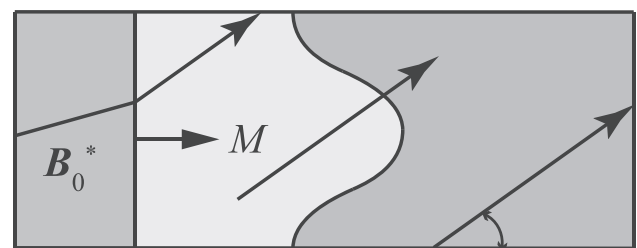

FIG. 1. (a) Geometry and initial condition for incompressible RMI with an external magnetic field $\boldsymbol{B}_{0}$ of arbitrary orientation. The perturbed density interface with wavelength $\Lambda$ and amplitude $\eta_{0}$ experiences impulsive acceleration $V_{0} \delta(t)$. (b) Two-dimensional incompressible RM flow representation. $\phi$ is the angle made by the in-plane background magnetic field with the z-axis. The hat symbol is used to denote dimensionless variables. (c) Initial conditions for the shock-driven compressible MHD RMI simulation. The asterisk symbol denotes a different non-dimensionalization scheme.

where $H(z)$ is the Heaviside function. This body force drives the flow and differs from the fictitious force $\rho \delta(t) \hat{z}$ in (1b) that accounts for the non-inertial reference frame. It is noted that in Eq. (2), $\rho_{1}=1$ and $\rho_{2}=\rho_{2} / \rho_{1}$ are indeed dimensionless. However, the notation of $\rho_{i=1,2}$ is kept here and henceforth in order to conveniently indicate the flow quantities of the two different fluids. Additionally, two dimensionless parameters are introduced, namely, the normalized Larmor radius $d_{L}$, and the plasma parameter $\beta$, defined as

$$
d_{L}=\frac{V_{0} m_{i}}{e B \Lambda}, \quad \beta=\frac{2 \mu_{0} \rho_{1} V_{0}^{2}}{B^{2}},
$$

where $\mu_{0}$ is the permeability of vacuum. The Larmor radius $d_{L}$ is the normal radius of the helix along which an ion moves about the background magnetic field lines, while the plasma parameter $\beta$ measures the ratio of kinetic to magnetic energy in an incompressible flow.

A distinguishing property of the Hall-MHD model is that by approximating the motion of electrons, its generalized Ohm's law (1d) contains the Hall term $(\boldsymbol{j} \times \boldsymbol{B}) / \rho$ and the electron pressure gradient component $d_{L} \nabla p_{e} / \rho{ }^{31}$ The Hall term generally introduces two wave modes into the plasma system: Whistler waves and Hall-drift waves. ${ }^{32,33}$ Under the current IIIE model where both ion and electron flows assume incompressibility, the Hall-drift effect is neglected. When substituted into the Faraday's law (1c) to give the Hall induction equation, the $\nabla p_{e} / \rho$ component in (1d) vanishes upon taking the curl in the flow regions of constant density. Shen et al. ${ }^{30}$ showed that although $p_{e}$ remains indeterminate in the IIIE model, retaining $\nabla p_{e} / \rho$ in (1d) constrains the electron pressure jump across the interface without affecting the flow dynamics. Also, using the present nondimensionalization scheme, the Hall-MHD system (1) reduces to the ideal MHD model in the continuous limit $d_{L} \rightarrow 0, \beta>0$. $^{31}$

\section{B. Rankine-Hugoniot conditions}

The boundary conditions for the contact discontinuity of simple geometry connecting the two flows on each side of the interface are given by the Rankine-Hugoniot conditions. These are derived by writing the governing equations (1) in conservation form and integrating over a shrinking volume that encloses the CD. For the present IIIE model, ${ }^{30}$ the CD jump conditions are

$$
\begin{gathered}
\llbracket u_{n} \rrbracket=0, \quad \llbracket \frac{j_{n}}{\rho} \rrbracket=0, \\
\llbracket\left(p+\frac{1}{\beta} B^{2}\right) \hat{\boldsymbol{n}}-\frac{2}{\beta} B_{n} \boldsymbol{B} \rrbracket=0, \\
\llbracket B_{n} \rrbracket=0,
\end{gathered}
$$

where the square brackets denote the difference between the two fluid regions on each side of the contact, and the subscript " $n$ " indicates the vector component normal to the surface.

\section{Linearized equations}

The base flow solution to (1), denoted by the bar symbol, corresponding to the impulsive acceleration of an unperturbed interface at $z=0$ is steady in the non-inertial frame, and given by

$$
\begin{aligned}
\overline{\boldsymbol{u}} & =\overline{\boldsymbol{j}}=\overline{\boldsymbol{E}}=\mathbf{0}, \quad \overline{\boldsymbol{B}}=\hat{\boldsymbol{B}}_{0}, \\
\bar{\rho}(z) & =\rho_{1}+H(z)\left(\rho_{2}-\rho_{1}\right), \quad \bar{p}(z, t)=p_{0},
\end{aligned}
$$

where $p_{0}$ is the constant background pressure, and the unit vector $\hat{\boldsymbol{B}}_{0}=\left(B_{0 x}, B_{0 y}, B_{0 z}\right)$ is three-dimensional in general.

For the perturbed interface, the density profile is expressed as

$$
\rho=\bar{\rho}(z-h),
$$

where $h(x, t)$ is the position of contact discontinuity and $h \ll 1$ is required to ensure a small perturbation for which linear theory applies. As a result, Eq. (1) can be linearized around the base flow by perturbing all flow fields using the form

$$
\xi(x, z, t)=\bar{\xi}(z)+\xi^{\prime}(x, z, t),
$$

where $\xi$ generically represents the scalar pressure $p$, or the vector components of $\boldsymbol{u}$ and $\boldsymbol{B} ; \xi^{\prime}$ is the corresponding perturbation of small 
magnitude (i.e., $\left|\xi^{\prime}\right| \ll|\bar{\xi}|$ ). In this construction, the perturbation wave is aligned in the $x$-direction so that no $y$-dependency is needed.

Using (1e) and (1d), both $\boldsymbol{j}$ and $\boldsymbol{E}$ are eliminated in favor of $\boldsymbol{B}$. The substitution of (7) then leads to the linearized equations

$$
\begin{gathered}
\frac{\partial u^{\prime}}{\partial x}+\frac{\partial w^{\prime}}{\partial z}=0 \\
\rho \frac{\partial u^{\prime}}{\partial t}+\frac{\partial p^{\prime}}{\partial x}=\frac{2}{\beta}\left[B_{0 z}\left(\frac{\partial B_{x}^{\prime}}{\partial z}-\frac{\partial B_{z}^{\prime}}{\partial x}\right)-B_{0 y} \frac{\partial B_{y}^{\prime}}{\partial x}\right] \\
\rho \frac{\partial v^{\prime}}{\partial t}=\frac{2}{\beta}\left(B_{0 z} \frac{\partial B_{y}^{\prime}}{\partial z}+B_{0 x} \frac{\partial B_{y}^{\prime}}{\partial x}\right) \\
\rho \frac{\partial w^{\prime}}{\partial t}+\frac{\partial p^{\prime}}{\partial z}+\frac{2}{\beta}\left[B_{0 x}\left(\frac{\partial B_{x}^{\prime}}{\partial z}-\frac{\partial B_{z}^{\prime}}{\partial x}\right)+B_{0 y} \frac{\partial B_{y}^{\prime}}{\partial z}\right] \\
=\left(\rho_{2}-\rho_{1}\right)[H(z)-H(z-h)] \delta(t) \\
\frac{\partial B_{x}^{\prime}}{\partial t}=B_{0 z} \frac{\partial u^{\prime}}{\partial z}+B_{0 x} \frac{\partial u^{\prime}}{\partial x}+\frac{2 d_{L}}{\beta \rho}\left(B_{0 z} \frac{\partial^{2} B_{y}^{\prime}}{\partial z^{2}}+B_{0 x} \frac{\partial^{2} B_{y}^{\prime}}{\partial x \partial z}\right) \\
-\frac{\partial v^{\prime}}{\partial z}+B_{0 x} \frac{\partial v^{\prime}}{\partial x}\left[B_{0 z} \frac{\partial}{\partial z}\left(\frac{\partial B_{x}^{\prime}}{\partial z}-\frac{\partial B_{z}^{\prime}}{\partial x}\right)+B_{0 x} \frac{\partial}{\partial x}\left(\frac{\partial B_{x}^{\prime}}{\partial z}-\frac{\partial B_{z}^{\prime}}{\partial x}\right)\right] \\
\frac{\partial B_{z}^{\prime}}{\partial t}=B_{0 z} \frac{\partial w^{\prime}}{\partial z}+B_{0 x} \frac{\partial w^{\prime}}{\partial x}-\frac{2 d_{L}}{\beta \rho}\left(B_{0 z} \frac{\partial^{2} B_{y}^{\prime}}{\partial x \partial z}+B_{0 x} \frac{\partial^{2} B_{y}^{\prime}}{\partial x^{2}}\right) \\
\frac{\partial B_{x}^{\prime}}{\partial x}+\frac{\partial B_{z}^{\prime}}{\partial z}=0
\end{gathered}
$$

where $\left(u^{\prime}, v^{\prime} w^{\prime}\right)$ and $\left(B_{x}^{\prime}, B_{y}^{\prime}, B_{z}^{\prime}\right)$ makeup the vectors $\boldsymbol{u}^{\prime}$ and $\boldsymbol{B}^{\prime}$, respectively. It is seen that the ideal MHD system is retrieved as a special case of the Hall-MHD system when $d_{L}=0$. $^{3}$

To proceed, we make the single Fourier-mode ansatz

$$
\xi^{\prime}(x, z, t)=\tilde{\xi}(z, t) e^{i k x},
$$

where $i$ is the imaginary unit and $k=2 \pi$ is the non-dimensional wavenumber since a fixed wavelength of $\Lambda$ (dimensional) is used for reference length. The contact is located at

$$
z=h(x, t)=\eta(t) e^{i k x}
$$

with $\eta(t)$ being the perturbation amplitude. It is required that $\eta \ll 1$ for any fixed orientation of $\hat{\boldsymbol{B}}_{0}$ so that the linear description (8) holds.

After the Fourier treatment, the temporal Laplace transform

$$
\mathcal{L}[\tilde{\xi}(t)]=\int_{0}^{\infty} \tilde{\xi}(t) e^{-s t} d t, \quad \Re(s)>0
$$

is further applied in the region $z<0$ and $z>h$, where the impulsive forcing vanishes. The initial conditions are taken at $t=0^{-}$, just prior to the impulse, when the velocity and magnetic field perturbations are zero. As a result, we obtain for each fluid in the region subscripted by $i=1,2$, a system of ordinary differential equations (ODEs) in the Laplace space given by

$$
i k U_{i}+\partial_{z} W_{i}=0
$$

$$
\begin{gathered}
s \rho_{i} U_{i}+i k P_{i}=\frac{2}{\beta}\left[B_{0 z}\left(\partial_{z} H_{x_{i}}-i k H_{z_{i}}\right)-i k B_{0 y} H_{y_{i}}\right], \\
s \rho_{i} V_{i}=\frac{2}{\beta}\left(B_{0 z} \partial_{z} H_{y_{i}}+i k B_{0 x} H_{y_{i}}\right), \\
s \rho_{i} W_{i}+\partial_{z} P_{i}+\frac{2}{\beta}\left[B_{0 x}\left(\partial_{z} H_{x_{i}}-i k H_{z_{i}}\right)+B_{0 y} \partial_{z} H_{y_{i}}\right]=0, \\
s H_{x_{i}}=B_{0 z} \partial_{z} U_{i}+i k B_{0 x} U_{i}+\frac{2 d_{L}}{\beta \rho_{i}}\left(B_{0 z} \partial_{z}^{2} H_{y_{i}}+i k B_{0 x} \partial_{z} H_{y_{i}}\right), \\
s H_{y_{i}}=B_{0 z} \partial_{z} V_{i}+i k B_{0 x} V_{i} \\
-\frac{2 d_{L}}{\beta \rho_{i}}\left[B_{0 z} \partial_{z}\left(\partial_{z} H_{x_{i}}-i k H_{z_{i}}\right)+i k B_{0 x}\left(\partial_{z} H_{x_{i}}-i k H_{z_{i}}\right)\right], \\
s H_{z_{i}}=B_{0 z} \partial_{z} W_{i}+i k B_{0 x} W_{i}-\frac{2 d_{L}}{\beta \rho_{i}}\left(i k B_{0 z} \partial_{z} H_{y_{i}}-k^{2} B_{0 x} H_{y_{i}}\right), \\
i k H_{x_{i}}+\partial_{z} H_{z_{i}}=0,
\end{gathered}
$$

where $U, V, W, H_{x}, H_{y}, H_{z}$, and $P$ are the Laplace transforms for $\tilde{u}, \tilde{v}, \tilde{w}, \tilde{B}_{x}, \tilde{B_{y}}, \tilde{B}_{z}$, and $\tilde{p}$, respectively.

\section{FLOW FIELD CALCULATION \\ A. Transverse magnetic field}

We first consider a special case of Eq. (12) where $B_{0 z}=0$, which corresponds to the background magnetic field being perpendicular to the initial impulsive acceleration, parallel to the $x, y$-plane.

\section{General solution}

The homogeneous system (12) evaluated at $B_{0 z}=0$ reduces to the following fourth-order ODE for $W_{i}$ that is independent of $B_{0 y}$ :

$$
\left(\frac{d^{2}}{d z^{2}}-k^{2}\right)\left(\frac{d^{2}}{d z^{2}}-\mu_{i}^{2}\right) W_{i}(z)=0,
$$

where

$$
\mu_{i}=\frac{\sqrt{4 k^{4}\left(d_{L}^{2} s^{2} B_{0 x}^{2}+B_{0 x}^{4}\right)+4 k^{2} B_{0 x}^{2} \beta \rho_{i} s^{2}+\beta^{2} \rho_{i}^{2} s^{4}}}{2 k B_{0 x} d_{L} s} .
$$

It is noted that in the ideal MHD limit $d_{L} \rightarrow 0$, the eigenvalue $\mu_{i}$ that appears in (13) escapes to infinity and the corresponding ODE for $W_{i}$ reduces to second order.

The desired general solution for $W_{i}$ to Eq. (13) must decay at $z= \pm \infty$, and therefore is given by

$$
W_{i}(z)=A_{i} e^{-k|z|}+B_{i} e^{-\mu_{i}|z|},
$$

where $A_{i}$ and $B_{i}$ are coefficients to be determined from appropriate interface boundary conditions. Once $W_{i}$ is known, other field variables follow directly from (12), including:

$$
\begin{aligned}
& H_{y_{i}}=\frac{i \beta d_{L} \rho_{i} s^{2}\left(k^{2} W_{i}-W_{i}^{\prime \prime}\right)}{2 k^{3} B_{0 x}^{2}+\beta k \rho_{i} s^{2}}, \quad H_{z_{i}}=\frac{B_{0 x}}{s \rho_{i}}\left(i k \rho_{i} W_{i}+\frac{2 d_{L} k^{2} H_{y_{i}}}{\beta}\right), \\
& H_{x_{i}}=\frac{-H_{z_{i}}{ }^{\prime}}{i k}, \quad U_{i}=\frac{-W_{i}^{\prime}}{i k}, \quad P_{i}=-\frac{\rho_{i} s W_{i}^{\prime}}{k^{2}}-\frac{2 B_{0 y} H_{y_{i}}}{\beta}
\end{aligned}
$$

where the prime symbol here denotes the derivative with respect to $z$. 


\section{Interface jump conditions}

Linearizing Eq. (4) around the baseflow given in (5) under the special consideration that the background magnetic field is parallel to the unperturbed $\mathrm{CD}$, i.e., $B_{0 z}=0$, leads to the following complete $\mathrm{CD}$ jump conditions in Laplace space:

$$
\begin{gathered}
\llbracket W \rrbracket_{z=0}=0,\left.\quad H_{y_{i}}\right|_{z=0}=0, \\
\llbracket P+\frac{2}{\beta}\left(B_{0 x} H_{x}+B_{0 y} H_{y}\right) \rrbracket_{z=0}=\eta_{0}\left(\rho_{2}-\rho_{1}\right), \\
\llbracket H_{z} \rrbracket_{z=0}=0,
\end{gathered}
$$

where the notation $\llbracket \xi(z) \rrbracket_{z=0}=\xi_{2}(0)-\xi_{1}(0)$ is used.

In particular, Eq. (17a) follows from the continuity condition (4a); and Eq. (17b) is obtained by evaluating (4b) at the interface $[z=h(x, t)]$ to give the leading order relation

$$
\begin{aligned}
& \tilde{p}_{2}(h, t)+\frac{2}{\beta}\left[B_{0 x} \tilde{B}_{x_{2}}(h, t)+B_{0 y} \tilde{B}_{y_{2}}(h, t)\right] \\
& \quad=\tilde{p}_{1}(h, t)+\frac{2}{\beta}\left[B_{0 x} \tilde{B}_{x_{1}}(h, t)+B_{0 y} \tilde{B}_{y_{1}}(h, t)\right]
\end{aligned}
$$

and integrating Eq. (8d) over the forcing region, $0<z<h(x, t)$, neglecting the terms of order $O\left(h^{2}\right)$ to yield

$$
\begin{aligned}
& {\left[\tilde{p}_{1}(z, t)+\frac{2}{\beta}\left(B_{0 x} \tilde{B}_{x_{1}}(z, t)+B_{0 y} \tilde{B}_{y_{1}}(z, t)\right)\right]_{z=0}^{z=h}} \\
& \quad=\left(\rho_{2}-\rho_{1}\right) \delta(t) \eta(t) .
\end{aligned}
$$

With $\tilde{p}_{2}, \tilde{B}_{x_{2}}$ and $\tilde{B}_{y_{2}}$ being smooth functions of $z$, substituting (18) into (19) and taking the temporal Laplace transform then produces (17b).

\section{Oscillatory solutions}

The unknown coefficients $A_{i}$ and $B_{i}$ can now be uniquely determined by solving the linear system obtained from substituting Eqs. (15) and (16) into (17).

It is also seen from (17) and (16) that the value of $B_{0 y}$ does not affect the final solution for $A_{i}$ and $B_{i}$. In fact, rotating the background magnetic field in the $x, y$-plane is equivalent to an effective change in $\beta$ that matches the corresponding field strength in the $x$ direction, $B_{0 x}$, while the $y$-component, $B_{0 y}$, has no effect on the flow dynamics. Thus, it is sufficient to set $B_{0 y}=0$ and $B_{0 x}=1$ without loss of generality. As a result, the final expression for $A_{i}$ and $B_{i}$ follows:

$$
A_{1}=A_{2}=\frac{\left(\rho_{2}-\rho_{1}\right) \beta \eta_{0} k s}{4 k^{2}+\beta \rho_{1} s^{2}+\beta \rho_{2} s^{2}}, \quad B_{1}=B_{2}=0 .
$$

Surprisingly, the eigenvalues $\mu_{i}$ associated with the Hall-MHD model found in (14) where $d_{L}>0$, does not enter the solution. Indeed, taking the inverse Laplace transform of Eq. (15) leads to the normal flow velocity, and hence via integration the interfacial perturbation amplitude that is identical to the MHD solution given by Wheatey et al. ${ }^{27}$

$$
\tilde{w}_{1,2}(z, t)=\eta_{0} k \mathcal{A} \cos (\omega t) e^{-k|z|},
$$

$$
\eta(t)=\eta_{0}+\int_{0^{+}}^{t} \tilde{w}_{1,2}(0, \tau) d \tau=\eta_{0}+\frac{\eta_{0} k \mathcal{A}}{\omega} \sin (\omega t)
$$

where $\mathcal{A} \equiv\left(\rho_{2}-\rho_{1}\right) /\left(\rho_{2}+\rho_{1}\right)$ is the Atwood number, and

$$
\omega \equiv \frac{2 k}{\sqrt{\beta\left(\rho_{1}+\rho_{2}\right)}}=\frac{k}{\sqrt{\frac{1}{2}\left(c_{A_{1}}^{-2}+c_{A_{2}}^{-2}\right)}}
$$

is the oscillation frequency expressed in terms of the Alfvén speeds $c_{A_{i}} \equiv \sqrt{2 /\left(\beta \rho_{i}\right)}$. In addition, a tangential slip velocity across the interface follows from (16) in this case, giving

$$
\Delta \tilde{u}(t) \equiv \tilde{u}_{2}(0, t)-\tilde{u}_{1}(0, t)=-2 i \eta_{0} k \mathcal{A} \cos (\omega t) .
$$

It is concluded that in the special case where the background magnetic field is aligned with the mean interfacial location, the Hall-MHD model predicts the same flow dynamics given by the ideal MHD theory. This is because the required $\mathrm{CD}$ jump conditions are uniquely satisfied by the eigenmodes corresponding to the Alfvén waves alone.

\section{B. Oblique magnetic field}

Now we generalize to an arbitrary oblique orientation of the background magnetic field, particularly allowing a component that is normal to the unperturbed interface, i.e., $B_{0 z}>0$.

\section{General solution}

To proceed, Eq. (12) is rearranged into a system of first order homogeneous ODEs by eliminating $P_{i}, V_{i}$, and $H_{z_{i}}$, yielding

$$
\frac{d y_{i}}{d z}=A_{i} y_{i},
$$

where

$$
\boldsymbol{y}_{i}=\left(H_{y_{i}}, \frac{d H_{y_{i}}}{d z}, H_{x_{i}}, \frac{d H_{x_{i}}}{d z}, U_{i}, W_{i}\right)^{\mathrm{T}}
$$

and $A_{i}$ is a $6 \times 6$ matrix whose elements are listed in Table I, Appendix A.

The general solution to Eq. (25) is therefore given by a linear combination of eigenmodes of the matrix $A_{i}$ as follows:

$$
\boldsymbol{y}_{i}(z)=\sum_{j=1}^{6} \alpha_{i, j} \exp \left(\lambda_{i, j} z\right) \boldsymbol{v}_{i, j}
$$

where $\alpha_{i, j}$ are unknown coefficients to be determined from the CD jump conditions; $\lambda_{i, j}$ is the eigenvalue for the fluid region $i$ that corresponds to the $j$-th root of the following polynomial of $\lambda$ :

$$
\begin{gathered}
4\left(k^{2}-\lambda^{2}\right)\left(k B_{0 x}-i \lambda B_{0 z}\right)^{2}\left[s^{2} d_{L}^{2}\left(k^{2}-\lambda^{2}\right)+\left(k B_{0 x}-i \lambda B_{0 z}\right)^{2}\right] \\
+\left(k^{2}-\lambda^{2}\right)\left[4 \beta s^{2} \rho_{i}\left(k B_{0 x}-i \lambda B_{0 z}\right)^{2}+\beta^{2} s^{4} \rho_{i}^{2}\right]=0
\end{gathered}
$$

and $\boldsymbol{v}_{i, j}$ is the corresponding eigenvector whose components are given in Table II, Appendix A.

Since the eigenvalues can be grouped by their signature, i.e.

$$
\Re\left(\lambda_{i, 1}\right), \Re\left(\lambda_{i, 2}\right), \Re\left(\lambda_{i, 3}\right)<0 ; \quad \Re\left(\lambda_{i, 4}\right), \Re\left(\lambda_{i, 5}\right), \Re\left(\lambda_{i, 6}\right)>0
$$

the boundedness of solution at $z= \pm \infty$ immediately requires that

$$
\alpha_{1,1}=\alpha_{1,2}=\alpha_{1,3}=\alpha_{2,4}=\alpha_{2,5}=\alpha_{2,6}=0 .
$$


Furthermore, once $y_{i}$ is determined, the remaining variables follow directly from (12), giving:

$$
\begin{gathered}
H_{z_{i}}=\frac{B_{0 z}}{s} \frac{d W_{i}}{d z}+\frac{i k B_{0 x}}{s} W_{i}-\frac{2 d_{L}}{\beta \rho_{i} s}\left(i k B_{0 z} \frac{d H_{y_{i}}}{d z}-k^{2} B_{0 x} H_{y_{i}}\right), \\
V_{i}=\frac{2}{\beta \rho_{i} s}\left(B_{0 z} \frac{d H_{y_{i}}}{d z}+i k B_{0 x} H_{y_{i}}\right), \\
P_{i}=\frac{-i}{k}\left[\frac{2}{\beta}\left(B_{0 z}\left(\frac{d H_{x_{i}}}{d z}-i k H_{z_{i}}\right)-i k B_{0 y} H_{y_{i}}\right)-s \rho_{i} U_{i}\right] .
\end{gathered}
$$

It is worth noting that the eigenvalue system (25) and hence its general solution (27) are independent of $B_{0 y}$, the out-of-plane component of the background field. However, the total pressure derived in (31c) does contain the term proportional to $B_{0 y} H_{y_{i}}$, same as in the transverse field case [see Eq. (16)].

\section{Modified $C D$ jump conditions}

Because an oblique magnetic field is applied in this section, the appropriate $\mathrm{CD}$ jump conditions must be modified in order to determine the remaining six unknown $\alpha_{i, j}$. It follows from Eq. (4) that when the magnetic field is not parallel to the interface, i.e., $B_{n} \neq 0$, both the pressure, $p$, and magnetic field, $\boldsymbol{B}$, must be continuous across the CD. Consequently, the linearized CD jump conditions in the Laplace space follow:

$$
\begin{gathered}
\llbracket W \rrbracket_{z=0}=0, \\
\llbracket H_{z} \rrbracket_{z=0}=0, \\
\llbracket H_{x} \rrbracket_{z=0}, \\
H_{y_{1}}(0)=H_{y_{2}}(0)=0, \\
\llbracket P \rrbracket_{z=0}=\eta_{0}\left(\rho_{2}-\rho_{1}\right),
\end{gathered}
$$

where continuity of $\tilde{p}, \tilde{H}_{x}$, and $\tilde{H}_{y}$ at the interface $z=h$ is used in Eq. (19) in order to arrive at (32e).

Again, as in Sec. III A 2, here Eqs. (31c)-(32e) imply that the effect of finite $B_{0 y}$ can be entirely captured by changing $\beta$. Therefore, we proceed with an in-plane background field

$$
\hat{\boldsymbol{B}}_{0}=(\sin \phi, 0, \cos \phi),
$$

where $\phi \in[0, \pi / 2)$ due to symmetry is the angle made by the applied field with the $z$-axis, as shown in Fig. 1(b). Thereafter, substituting (27), (31), and (33) into (32) produces a set of linear equations from which the indeterminate $\alpha_{i, j}$ can be uniquely solved. The resulting expressions are cumbersome and therefore omitted for brevity.

It is important to note that forcing continuity of the tangential magnetic field as well as the pressure in (32c)-(32e) suggests that the "effective pressure" condition (17b) required in the transverse field case is also satisfied when an oblique field applies, making Eq. (32) a subset of Eq. (17). This means the solution space available for (17) is expanded since more eigenmodes are included. The significance of having a larger solution space is further discussed in Sec. IV D, where the limiting flow as $\phi \rightarrow \pi / 2$ is considered.

\section{Interface response}

Having obtained the flow solution in the Laplace space, the temporal behavior is determined through the inverse transform formally given by the Bromwich integral

$$
\mathcal{L}^{-1}[G(s)]=\frac{1}{2 \pi i} \int_{r-i \infty}^{r+i \infty} G(s) e^{t s} d s,
$$

where $r \in \mathbb{R}$ is greater than any real part of the singularities of the function $G(s)$. In particular, the interface perturbation amplitude is calculated as

$$
\eta(t)=\eta_{0}+\mathcal{L}^{-1}\left[\frac{W(0 ; s)}{s}\right] .
$$

Additionally, circulation deposition at the interface due to tangential slip velocity is facilitated by the Hall-MHD description when an oblique magnetic field is present. To leading order, this circulation over a half wavelength (recalling $\Lambda=1, k=2 \pi$ ) is given by

$$
\gamma_{0}(t)=\mathcal{L}^{-1}\left[\Delta U \int_{0}^{\Lambda / 2} e^{i k x} d x\right]=\mathcal{L}^{-1}\left[\frac{2 i \Delta U}{k}\right],
$$

where $\Delta U \equiv U_{2}(0)-U_{1}(0)$. It can be verified that the initial interface behavior is dictated by the pure hydrodynamic response, independent of the strength and orientation of the applied magnetic field, i.e.

$$
\left.\frac{d \eta}{d t}\right|_{t=0^{+}}=\eta_{0} k \mathcal{A}, \quad \gamma_{0}\left(0^{+}\right)=4 \eta_{0} \mathcal{A} .
$$

\section{Vorticity transport}

The lack of a transport mechanism for vorticity, $\boldsymbol{\omega}=\nabla \times \boldsymbol{u}$, after its initial baroclinic generation is the essential cause of the RMI in a non-conducting gas. ${ }^{1}$ However in a plasma described by the HallMHD model, vorticity is governed by

$$
\frac{\partial \boldsymbol{\omega}}{\partial t}+(\boldsymbol{u} \cdot \nabla) \boldsymbol{\omega}=(\boldsymbol{\omega} \cdot \nabla) \boldsymbol{u}+\frac{\nabla \rho \times \nabla p}{\rho^{2}}+\frac{2}{\beta \rho}[\nabla \times(\nabla \times \boldsymbol{B} \times \boldsymbol{B})],
$$

where the magnetic field clearly affects the dynamics. Linearizing (38) around the base-flow discussed in Sec. II C subject to an oblique magnetic field in the $x, z$-plane produces

$$
\frac{\partial \tilde{\boldsymbol{\omega}}}{\partial t}=\frac{2}{\beta \rho_{i}}\left[\begin{array}{c}
-\cos \phi \frac{\partial^{2} \tilde{B}_{y}}{\partial z^{2}}-i k \sin \phi \frac{\partial \tilde{B}_{y}}{\partial z} \\
\cos \phi\left(\frac{\partial^{2} \tilde{B}_{x}}{\partial z^{2}}-i k \frac{\partial \tilde{B}_{z}}{\partial z}\right)+\sin \phi\left(i k \frac{\partial \tilde{B}_{x}}{\partial z}+k^{2} \tilde{B}_{z}\right) \\
i k \cos \phi \frac{\partial \tilde{B}_{y}}{\partial z}-k^{2} \sin \phi \tilde{B}_{y}
\end{array}\right]
$$

for the linearized vorticity vector

$$
\tilde{\boldsymbol{\omega}}=\left(-\frac{\partial \tilde{v}}{\partial z},-i k \tilde{w}+\frac{\partial \tilde{u}}{\partial z}, i k \tilde{v}\right)^{\mathrm{T}} .
$$

Differentiating (39) with respect to time $t$ once more using (8) then implies

$$
\frac{\partial^{2} \tilde{\boldsymbol{\omega}}}{\partial t^{2}}=\frac{2}{\beta \rho_{i}} \mathcal{F}[\tilde{\boldsymbol{\omega}}]+\frac{4 d_{L}}{\beta^{2} \rho_{i}^{2}} \mathcal{F}\left[\frac{\partial^{2} \tilde{\boldsymbol{B}}}{\partial z^{2}}-k^{2} \tilde{\boldsymbol{B}}\right],
$$

where the operator $\mathcal{F}$ is given by

$$
\mathcal{F}=\cos ^{2} \phi \frac{\partial^{2}}{\partial z^{2}}+2 i k \cos \phi \sin \phi \frac{\partial}{\partial z}-k^{2} \sin ^{2} \phi .
$$


In view of $\partial / \partial x=i k, \mathcal{F}$ becomes

$$
\mathcal{F}=\left(\sin \phi \frac{\partial}{\partial x}+\cos \phi \frac{\partial}{\partial z}\right)^{2}=\frac{\partial^{2}}{\partial r^{2}},
$$

where $r$ is defined as the path length in the magnetic field direction

$$
x=r \sin \phi, \quad z=r \cos \phi .
$$

Therefore, Eq. (41) simplifies to a forced wave equation

$$
\frac{\partial^{2} \tilde{\boldsymbol{\omega}}}{\partial t^{2}}=\frac{2}{\beta \rho_{i}} \frac{\partial^{2} \tilde{\boldsymbol{\omega}}}{\partial r^{2}}+\frac{4 d_{L}}{\beta^{2} \rho_{i}^{2}} \frac{\partial^{2}\left(\nabla^{2} \tilde{\boldsymbol{B}}\right)}{\partial r^{2}},
$$

where the normalized Alfvén waves propagate along the magnetic field lines at the speed of $c_{A_{i}}=\sqrt{2 /\left(\beta \rho_{i}\right)}$. For an oblique field angle $\phi$, a coupling between waves traveling parallel and normal to the interface is expected, leading to a continuous change of phase at the interface.

Equation (45) contains a vorticity source term, produced by magnetic field perturbations, that vanishes in the MHD limit $d_{L} \rightarrow 0$ for all $\phi$. The vorticity forcing in Hall-MHD is proportional to both the Larmor radius $d_{L}$ and the square of Alvén speed $c_{A}^{2}$, suggesting that vorticity production is generated by Whistler waves available in the Hall-MHD system whose frequencies also scale as $\omega \propto c_{A}^{2} / d_{L} \cdot{ }^{31,34}$ As a result, conservation of circulation following the initial baroclinic generation, that holds for a non-conducting gas and also for regular MHD, is no longer true in Hall-MHD.

\section{ASYMPTOTIC ANALYSIS}

In this section, we explore the limiting behavior of the impulsive RM flow subject to an oblique magnetic field obtained in Sec. III $B$, with respect to extreme values of the Larmor radius $d_{L}$, the plasma energy ratio $\beta$, and the field angle $\phi$. It is also insightful to discuss one more related parameter, the normalized ion skin depth $d_{S}$, given by

$$
d_{S}=d_{L} \sqrt{\frac{2}{\beta}}
$$

which is independent of the applied magnetic field. The plasma region beyond ideal MHD where $d_{S} \gg 1$ will be examined.

\section{A. The MHD limit}

Upon passing the limit $d_{L} \rightarrow 0$ with $\beta$ held fixed, to the general Hall-MHD solution developed previously, we immediately obtain the RM flow field corresponding to the ideal MHD model. It is verified that in such a limit, first, the out-of-plane components of the flow and magnetic fields, $V_{i}$ and $H_{y_{i}}$, decouple from the system (12); and second, the MHD Rankine-Hugoniot relations, which require that $u, w$, $B_{x}, B_{z}$, and $p$ are continuous across the contact, are uniformly satisfied by the Hall-MHD CD jump conditions (32). Indeed, as $d_{L} \rightarrow 0$, Eqs. (12a), (31a), and (32b) show that the tangential slip velocity vanishes, i.e., $\Delta U=0$.

\section{Derivation for perturbation velocity}

Nonetheless, physics of the limiting MHD flow is better understood by explicitly solving the corresponding boundary value problem. The solution to this problem was briefly presented in Ref. 35 and is treated in significantly more detail here. To proceed, the eigenvalue equation (28) factorizes as $d_{L} \rightarrow 0$, and (27) gives in particular

$$
\begin{aligned}
W_{i}= & A_{i} e^{k z}+B_{i} e^{-k z} \\
& +\left(C_{i} e^{s \sqrt{\beta \rho_{1} / 2} \sec \phi}+D_{i} e^{-s \sqrt{\beta \rho_{1} / 2} \sec \phi}\right) e^{-i k z \tan \phi},
\end{aligned}
$$

where $A_{2}=B_{1}=C_{2}=D_{1}=0$ for decaying modes. The factor of $e^{i k z \tan \phi}$ that multiplies the final two terms is the only modification to the general solution found by Wheatley et al..$^{25}$ for the normal magnetic field case.

The CD jump conditions (32) in the MHD limit translate into

$$
\begin{aligned}
& \llbracket W \rrbracket_{z=0}=\llbracket \partial_{z} W \rrbracket_{z=0}=\llbracket \partial_{z}^{2} W \rrbracket_{z=0}=0, \\
& \frac{2 \cos ^{2} \phi}{\beta} \llbracket \partial_{z}^{3} W \rrbracket_{z=0}-s^{2}\left(\left.\rho_{2} \partial_{z} W_{2}\right|_{z=0}\right. \\
& \left.-\left.\rho_{1} \partial_{z} W_{1}\right|_{z=0}\right)=s k^{2}\left(\rho_{2}-\rho_{1}\right) \eta_{0} .
\end{aligned}
$$

Substituting (47) into (48) and solving simultaneously for the coefficients then produces

$$
\begin{aligned}
& A_{1}=k \eta_{0} \mathcal{A} \frac{s\left(s+\bar{f}_{1}\right)}{\left(s-f_{1}\right)(s-\theta)(s-\bar{\theta})}, \\
& B_{2}=k \eta_{0} \mathcal{A} \frac{s\left(s+f_{2}\right)}{\left(s-\bar{f}_{2}\right)(s-\theta)(s-\bar{\theta})}, \\
& C_{1}=-\frac{2 \sqrt{2} \cos \phi k^{2} \eta_{0} \mathcal{A}}{\sqrt{\beta \rho_{1}}+\sqrt{\beta \rho_{2}}} \sqrt{\frac{\rho_{2}}{\rho_{1}}} \frac{s+f_{2}}{\left(s-f_{1}\right)(s-\theta)(s-\bar{\theta})}, \\
& D_{2}=-\frac{2 \sqrt{2} \cos \phi k^{2} \eta_{0} \mathcal{A}}{\sqrt{\beta \rho_{1}}+\sqrt{\beta \rho_{2}}} \sqrt{\frac{\rho_{1}}{\rho_{2}}} \frac{s+\bar{f}_{1}}{\left(s-\bar{f}_{2}\right)(s-\theta)(s-\bar{\theta})} .
\end{aligned}
$$

Here, the poles of the above functions are defined using

$$
\theta=\kappa+i \omega, \quad f_{j}=f_{z_{j}}+i f_{x_{j}} \quad(j=1,2),
$$

where

$$
\begin{aligned}
\kappa & =-\cos \phi \sqrt{\frac{2}{\beta}} k \frac{\sqrt{\rho_{1}}+\sqrt{\rho_{2}}}{\rho_{1}+\rho_{2}} \\
\omega & =k\left[\frac{4 \sin ^{2} \phi}{\beta\left(\rho_{1}+\rho_{2}\right)}+\frac{2}{\beta}\left(\frac{\cos \phi\left(\sqrt{\rho_{2}}-\sqrt{\rho_{1}}\right)}{\rho_{1}+\rho_{2}}\right)^{2}\right]^{1 / 2}, \\
f_{x_{j}} & =c_{A_{j} x} k, \quad f_{z_{j}}=c_{A_{j} z} k
\end{aligned}
$$

and $\boldsymbol{c}_{A_{j}}=\left(c_{A_{j} x}, c_{A_{j} z}\right)=\hat{\boldsymbol{B}}_{0} \sqrt{2 /\left(\beta \rho_{j}\right)}$ is the Alfvén wave velocity in fluid $j$. The $z$-component velocity perturbations in each fluid are then obtained by taking the inverse Laplace transform of (47). This yields

$$
\begin{aligned}
w_{1}^{\prime}(x, z, t)= & \hat{a}_{1}(t) e^{k z+i k x}+\hat{c}_{1}\left(t+z / c_{A_{1} z}\right) \\
& \times e^{i k\left(x-z c_{A_{1} x} / c_{A_{1}} z\right.} H\left(t+z / c_{A_{1} z}\right), \\
w_{2}^{\prime}(x, z, t)= & \hat{b}_{2}(t) e^{-k z+i k x}+\hat{d}_{2}\left(t-z / c_{A_{2} z}\right) \\
& \times e^{i k\left(x-z c_{A_{2} x} / c_{A_{2} z}\right)} H\left(t-z / c_{A_{2} z}\right),
\end{aligned}
$$

where 


$$
\begin{aligned}
\hat{a}_{1}(t)= & k \eta_{0} \mathcal{A}\left\{\frac{2 f_{z_{1}} f_{1}}{\left(f_{1}-\theta\right)\left(f_{1}-\bar{\theta}\right)} e^{f_{1} t}\right. \\
& \left.+\frac{e^{\kappa t}}{2 i \omega}\left[\frac{\theta\left(\theta+\bar{f}_{1}\right)}{\theta-f_{1}} e^{i \omega t}-\frac{\bar{\theta}\left(\bar{\theta}+\bar{f}_{1}\right)}{\bar{\theta}-f_{1}} e^{-i \omega t}\right]\right\}, \\
\hat{b}_{2}(t)= & k \eta_{0} \mathcal{A}\left\{\frac{2 f_{z_{2}} \bar{f}_{2}}{\left.\left(\bar{f}_{2}-\theta\right)(\bar{f})_{2}-\bar{\theta}\right)} e^{\bar{f}_{2} t}\right. \\
& \left.+\frac{e^{\kappa t}}{2 i \omega}\left[\frac{\theta\left(\theta+f_{2}\right)}{\theta-f_{2}} e^{i \omega t}-\frac{\bar{\theta}\left(\bar{\theta}+f_{2}\right)}{\bar{\theta}-f_{2}} e^{-i \omega t}\right]\right\}, \\
\hat{c}_{1}(t)= & -\frac{2 \sqrt{2} \cos \phi k^{2} \eta_{0} \mathcal{A}}{\sqrt{\beta \rho_{1}}+\sqrt{\beta \rho_{2}}} \sqrt{\frac{\rho_{2}}{\rho_{1}}}\left\{\frac{f_{1}+f_{2}}{\left(f_{1}-\theta\right)\left(f_{1}-\bar{\theta}\right)} e^{f_{1} t}\right. \\
& \left.+\frac{e^{\kappa t}}{2 i \omega}\left[\frac{\theta+f_{2}}{\theta-f_{1}} e^{i \omega t}-\frac{\bar{\theta}+f_{2}}{\bar{\theta}-f_{1}} e^{-i \omega t}\right]\right\}, \\
\hat{d}_{2}(t)= & -\frac{2 \sqrt{2} \cos \phi k^{2} \eta_{0} \mathcal{A}}{\sqrt{\beta \rho_{1}}+\sqrt{\beta \rho_{2}}} \sqrt{\frac{\rho_{1}}{\rho_{2}}}\left\{\frac{\bar{f}_{1}+\bar{f}_{2}}{\left(\bar{f}_{2}-\theta\right)\left(\bar{f}_{2}-\bar{\theta}\right)} e^{\bar{f}_{2} t}\right. \\
& \left.+\frac{e^{\kappa t}}{2 i \omega}\left[\frac{\theta+\bar{f}_{1}}{\theta-\bar{f}_{2}} e^{i \omega t}-\frac{\bar{\theta}+\bar{f}_{1}}{\bar{\theta}-\bar{f}_{2}} e^{-i \omega t}\right]\right\} .
\end{aligned}
$$

Here, the term involving $\hat{a}_{1}(t)$ arises from the inverse Laplace transform of $A_{1}(s)$ and so forth. It can be verified that substituting $\phi=$ $\pi / 2$ into (52) recovers Eq. (21), the transverse magnetic field solution discussed in Sec. III A.

\section{Wave propagation}

The Heaviside functions that appear in the solution (52) correspond to finite-amplitude, non-uniform Alfvén waves, which we refer to as Alfvén fronts. These propagate outward from the interface at the relevant Alfvén speeds. Such waves are permitted in an incompressible flow since density and normal velocity are continuous across them, as are pressure and normal magnetic field. The finite amplitude waves divide the solution into four regions: $z<-c_{A_{1} z} t$, where $w^{\prime}=\hat{a}_{1}(t) e^{k z+i k x} ;-c_{A_{1} z} t<z<0$, where both $\hat{a}_{1}$ and $\hat{c}_{1}$ terms contribute to $w^{\prime} ; 0<z<c_{A_{2} z} t$, where $w^{\prime}$ is given by terms involving $\hat{b}_{2}$ and $\hat{d}_{2}$; and $z>c_{A_{2} z} t$, where $w^{\prime}=\hat{b}_{2}(t) e^{-k z+i k x}$. Observe that the solution below the interface is equivalent to that above, but with $\rho_{1}$ and $\rho_{2}$ interchanged and the dependence on $z$ reversed. It is therefore sufficient to discuss the features of the solution for $z>0$. The leading term in $\hat{b}_{2}(t) e^{-k z+i k x}$ has the form $K_{b} e^{-k\left(z-c_{A_{2}} t\right)+i k\left(x-c_{A_{2} x} t\right)}$, which is wave-like in nature, propagating away from the density interface at the Alfvén speed parallel to the base magnetic field. This mode decays exponentially upstream of the upper Alfvén front and grows exponentially downstream $\left(0<z<c_{A_{2}} t\right)$. However, exponential growth in $w^{\prime}$ does not occur because in this region the leading terms arising from $\hat{b}_{2}$ and $\hat{d}_{2}$ can be shown to cancel. The remaining terms arising from $\hat{b}_{2}$ correspond to oscillations at angular frequency $\omega$ that decay exponentially in time since $\kappa<0$. The oscillations also decay exponentially with distance above the interface like $e^{-k z}$. The mode arising from $\hat{d}\left(t-z / c_{A_{2} z}\right)$ is entirely wave-like in nature and propagates parallel to the base magnetic field. This mode, which due to the Heaviside function is only present between the upper Alfvén front and the interface, introduces a gradient discontinuity in $w^{\prime}$ at the front, which from (12a) implies a tangential velocity discontinuity. This results in the Alfvén front, and its partner in the $z<0$ fluid, transporting the vorticity generated by the impulsive acceleration of the interface from its location. Thus, the requirement that the tangential velocity slip across the CD be zero in the MHD limit is satisfied for $t>0$. Since the distribution of vorticity induces the interfacial velocities responsible for perturbation growth, its transport from the interface on the Alfvén fronts is the mechanism that underpins mitigation of the MHD RMI when a magnetic field is present.

\section{B. Large skin depth limit}

Shen et al. ${ }^{31}$ showed that the ideal MHD equations correspond to the formal limit of small skin depth, $d_{S}$, of the Hall-MHD system. Therefore, complementary to the MHD theory, the opposite limit of $d_{S} \rightarrow \infty$, is examined next. This limit manifests the strong effect of the Hall current. ${ }^{34}$ According to (46), the limit can be achieved by either taking $\beta \rightarrow 0$ with $d_{L}$ held finite, or letting $d_{L} \rightarrow \infty$ while keeping $\beta$ fixed. Both cases are shown to produce the same result in the following.

\section{Limit approached with small $\beta$}

Using the quartic root formula, the eigenvalues found in (28) can be Taylor expanded around $\beta=0$ to first order, giving

$$
\begin{aligned}
\lambda= & \pm k, \pm\left[\frac{\beta \rho_{i} s}{2 k d_{L} \sqrt{\sigma^{2}+1}}+\frac{k\left(\sigma \sqrt{\sigma^{2}+1} \mp i \sin \phi \cos \phi\right)}{\sigma^{2}+\cos ^{2} \phi}\right], \\
& \pm \frac{\beta \rho_{i} s}{2 k d_{L}}-i k \tan \phi
\end{aligned}
$$

where $\sigma=d_{L} s$.

Substituting the general solutions (27) and (31) with approximated eigenvalues and eigenvectors into (32) yields a system of linear equations [see Eq. (B1), Appendix B] from which the coefficients $\alpha_{i, j}$ are solved to yield

$$
\alpha_{2,2}=\alpha_{1,5}=\frac{\eta_{0} k \mathcal{A} d_{L}}{1+\sqrt{1+\sigma^{2}}}, \quad \alpha_{2,1}=\alpha_{2,3}=\alpha_{1,4}=\alpha_{1,6}=0 .
$$

Consequently, one obtains from (27) the transformed in-plane flow field

$$
\begin{gathered}
W_{1,2}(z)=\frac{\eta_{0} \mathcal{A} k d_{L}}{1+\sqrt{1+\sigma}} \exp \left(\chi^{ \pm} z\right), \\
U_{1,2}(z)= \pm \frac{i \eta_{0} \mathcal{A} k d_{L}\left(\sigma \sqrt{1+\sigma^{2}} \mp i \sin \phi \cos \phi\right)}{\left(1+\sqrt{1+\sigma^{2}}\right)\left(\sigma^{2}+\cos ^{2} \phi\right)} \exp \left(\chi^{ \pm} z\right),
\end{gathered}
$$

where

$$
\chi^{ \pm}= \pm \frac{k\left(\sigma \sqrt{\sigma^{2}+1} \mp i \sin \phi \cos \phi\right)}{\sigma^{2}+\cos ^{2} \phi} .
$$

Inspired by the definition of $\sigma$, it is convenient to renormalize time $t$ accordingly and define the ion cyclotron timescale $T \equiv t / d_{L} \cdot{ }^{30}$ Using (35) and (36), inverting the Laplace transform $\left(\mathcal{L}^{-1}: \sigma \rightarrow T\right)$ thus leads to 


$$
\begin{gathered}
\frac{\hat{\eta}-\hat{\eta}_{0}}{d_{L}}=T_{1} F_{2}\left(-\frac{1}{2} ; 1, \frac{3}{2} ;-\frac{T^{2}}{4}\right)-\frac{T^{2}}{2}, \\
\hat{\gamma}_{0}=\frac{\left(\cos ^{2} \phi-1\right) \cos (T \cos \phi)-\cos \phi \sin (T \cos \phi)+1}{\cos ^{2} \phi} \\
-\frac{1-\cos (T \cos \phi)}{\cos ^{2} \phi} * \frac{J_{1}(T)}{T},
\end{gathered}
$$

where $\hat{\eta}=\eta /\left(\mathcal{A} \eta_{0} k\right), \hat{\gamma}_{0}=\gamma_{0} /\left(4 \eta_{0} \mathcal{A}\right),{ }_{1} F_{2}$ is the generalized hypergeometric function, ${ }^{36} J_{1}$ is the first order Bessel function of the first kind, and $*$ denotes the convolution integral given by

$$
f(T) * g(T)=\int_{0}^{T} f(\tau) g(T-\tau) d \tau .
$$

The detailed steps of Laplace transform inversion toward (59) and (60) is provided in Appendix C. Interestingly, the growth of the interface perturbation found in (59) is independent of the field angle $\phi$, but the circulation deposition derived in (60) is not. In this case, the flow fields away from the CD must adjust according to the varying circulation as $\phi$ changes, so that a common interfacial growth is maintained.

\section{Limit approached with large $\mathrm{d}_{\mathrm{L}}$}

The alternative route to access the large $d_{S}$ region is realized in the dual Laplace space, $(s, t) \mapsto(\sigma, T)$, and applying the distinguished limit of $t \rightarrow \infty$ while holding $T$ constant. Specifically, the eigenvalues as functions of $\sigma$ are first expanded in the power series of $\epsilon \equiv 1 / d_{L}$ around $\epsilon=0$, giving

$$
\begin{aligned}
\lambda= & \pm k, \pm \frac{\beta \rho_{i} \sigma \epsilon^{2}}{2 k}-i k \tan \phi, \\
& \pm \frac{\sigma}{2 k \sqrt{\sigma^{2}+1}}\left(\beta \rho_{i} \epsilon^{2}+\frac{2 k^{2}\left(\sigma^{2}+1\right)}{\sigma^{2}+\cos ^{2} \phi}\right)-\frac{i k \sin \phi \cos \phi}{\sigma^{2}+\cos ^{2} \phi} .
\end{aligned}
$$

Again, applying the boundary conditions to the approximated eigenmodes [see Eq. (B3), Appendix B] leads to $\alpha_{i, j}$ in the limit

$$
\alpha_{2,2}=\alpha_{1,5}=\frac{\eta_{0} k \mathcal{A}}{\left(1+\sqrt{1+\sigma^{2}}\right) \epsilon}, \quad \alpha_{2,1}=\alpha_{2,3}=\alpha_{1,4}=\alpha_{1,6}=0
$$

which is effectively identical to the previous result (55). It immediately follows that the flow field in the present $d_{L} \rightarrow \infty$ limit, viewed in the cyclotron timescale, is the same as those given in Eqs. (56)-(60). We therefore distinctly attribute such a flow field to the common large ion skin depth limit, obtained as $d_{S} \rightarrow \infty$.

\section{Strong field limit}

Here, we briefly discuss the flow region where the hydrodynamic forces are dominated by a large magnetic field. Since $d_{S}$ is not a function of the applied field strength, this region corresponds to the limit of $\beta \rightarrow 0$ while holding $d_{S}$ fixed. In this case, all six eigenvalues take the asymptotic form of

$$
\lambda \sim-i k \tan \phi+O\left(\beta^{1 / 2}\right)
$$

leading to uniform decay of the coefficients $\alpha_{i, j}$ as

$$
\alpha_{2,1} \sim \alpha_{1,6} \sim O(\beta), \quad \alpha_{2,2} \sim \alpha_{2,3} \sim \alpha_{1,4} \sim \alpha_{1,5} \sim O\left(\beta^{1 / 2}\right) .
$$

Hence, from Eq. (3), increasing the external magnetic field strength inhibits any perturbation to the base-flow, as all variables in (27) and (31) decay asymptotically as follows:

$$
W, V \sim O\left(\beta^{1 / 2}\right), \quad U \sim\left\{\begin{array}{ll}
O\left(\beta^{1 / 2}\right), & \phi>0 \\
O(\beta), & \phi=0
\end{array}, \quad H_{x, y, z} \sim O(\beta)\right.
$$

and the normal flow is always inversely proportional to the applied field strength. In the limit $\beta \rightarrow 0$, the initial impulse propagates in time with a frozen interface, due to an overwhelming background magnetic field.

\section{Large angle limit}

The last limit of interest occurs when the background magnetic is nearly parallel to the mean interface, namely, $\phi \rightarrow \pi / 2$, while maintaining a small component in the normal direction. The flow field is approached in this case by expansions around $\varepsilon=0$, after substituting $B_{0 z}=\varepsilon$, and $B_{0 x}=\sqrt{1-\varepsilon^{2}}$. For the linear theory developed in Sec. II $C$ to be valid under this limit, it is required that the perturbation magnitude is sufficiently small, i.e., $\eta \ll \varepsilon \ll 1$.

To proceed, the applicable eigenvalues are those obtained in (28) for an oblique magnetic field, approximated by

$$
\lambda= \pm k, \pm \mu_{i}^{*}-\frac{i \varepsilon\left(4 k^{4}-\beta^{2} \rho_{i}^{2} s^{4}\right)}{4 k^{3} d_{L}^{2} s^{2}}, \pm \frac{\beta \rho_{i} s}{2 k d_{L}}+\frac{i k\left(\varepsilon^{2}-2\right)}{2 \varepsilon}-\frac{i \beta^{2} \rho_{i}^{2} s^{2} \varepsilon}{4 k^{3} d_{L}^{2}},
$$

where $\mu_{i}^{*}$ is a special value of $\mu_{i}$, defined in (14), evaluated at $B_{0 x}=1$. It is observed that as $\varepsilon \rightarrow 0$, two of the eigenvalues diverge, in addition to recovering those associated with the transverse field case found in (13). As a result, all six eigenmodes are utilized in order to meet the oblique field jump conditions (32), generating the following coefficients from Eq. (B5), Appendix B, at order $O\left(\varepsilon^{0}\right)$ :

$$
\begin{aligned}
& \alpha_{2,1}=\frac{\beta \eta_{0} k\left(\rho_{1}-\rho_{2}\right) s\left(4 k^{4} d_{L}\left(\rho_{1}-\rho_{2}\right) s-\psi\right)}{4 \beta k^{4} d_{L}\left(\rho_{1}-\rho_{2}\right)^{2} s^{3}+4 k^{2} \psi+\beta\left(\rho_{1}+\rho_{2}\right) s^{2} \psi}, \\
& \alpha_{2,2}=\frac{-8 \eta_{0} k^{5} d_{L}\left(\rho_{1}-\rho_{2}\right)\left(2 k^{2}+\beta \rho_{1} s^{2}\right)}{4 \beta k^{4} d_{L}\left(\rho_{1}-\rho_{2}\right)^{2} s^{3}+4 k^{2} \psi+\beta\left(\rho_{1}+\rho_{2}\right) s^{2} \psi}, \\
& \alpha_{1,5}=\frac{-8 \eta_{0} k^{5} d_{L}\left(\rho_{1}-\rho_{2}\right)\left(2 k^{2}+\beta \rho_{2} s^{2}\right)}{4 \beta k^{4} d_{L}\left(\rho_{1}-\rho_{2}\right)^{2} s^{3}+4 k^{2} \psi+\beta\left(\rho_{1}+\rho_{2}\right) s^{2} \psi}, \\
& \alpha_{1,6}=\frac{\beta \eta_{0} k\left(\rho_{1}-\rho_{2}\right) s\left(4 k^{4} d_{L}\left(\rho_{2}-\rho_{1}\right) s-\psi\right)}{4 \beta k^{4} d_{L}\left(\rho_{1}-\rho_{2}\right)^{2} s^{3}+4 k^{2} \psi+\beta\left(\rho_{1}+\rho_{2}\right) s^{2} \psi}, \\
& \alpha_{2,3}=\alpha_{1,4}=0,
\end{aligned}
$$

where

$$
\begin{aligned}
\psi= & 2 k^{2} \rho_{2}\left(2 k^{2}+2 k s d_{L} \mu_{2}^{*}+\beta \rho_{2} s^{2}\right)+\beta \rho_{1}^{2} s^{2}\left(2 k^{2}+\beta \rho_{2} s^{2}\right) \\
& +\rho_{1}\left(4 k^{4}+4 k^{3} s d_{L} \mu_{1}^{*}+2 k \beta \rho_{2} s^{2}\left(2 k+s d_{L} \mu_{1}^{*}+s d_{L} \mu_{2}^{*}\right)+\beta^{2} \rho_{2}^{2} s^{4}\right) .
\end{aligned}
$$

\section{Comparison with the transverse field case}

The interface growth rate is calculated using (27) and (68) as 


$$
\begin{aligned}
& W_{1,2}(0) \\
& =\frac{\eta_{0} k\left(\rho_{2}-\rho_{1}\right)\left(16 k^{6} d_{L}+4 \beta k^{4} d_{L}\left(\rho_{1}+\rho_{2}\right) s^{2}+\beta s \psi\right)}{\beta s^{2}\left(\rho_{2}\left(4 k^{4} d_{L} \rho_{2} s+\psi\right)+\rho_{1}\left(\psi-8 k^{4} d_{L} \rho_{2} s\right)+4 k^{4} d_{L} \rho_{1}^{2} s\right)+4 k^{2} \psi}
\end{aligned}
$$

which obviously differs from the one derived for a strictly parallel magnetic field where $\phi=\pi / 2$, given in Sec. III A 3, rendering the latter a singular limit, as the eigenvalue equation (28) loses its term of highest power of $\lambda$ when $\varepsilon=0$. In addition, in Eq. (32), the CD jump conditions associated with an oblique background field do not converge to Eq. (17), its parallel field counterpart. Particularly, the magnetic field may be discontinuous at the interface when a strictly parallel background is present. Indeed, substituting (20) into (16) shows when $\phi=\pi / 2$

$$
\Delta H_{x} \equiv H_{x_{2}}(0)-H_{x_{1}}(0)=\frac{2 \eta_{0} k^{2} \beta\left(\rho_{2}-\rho_{1}\right)}{4 k^{2}+\beta\left(\rho_{1}+\rho_{2}\right) s^{2}} \neq 0 .
$$

However, as $\phi \rightarrow \pi / 2$ with $\varepsilon>0$, using (27) and (67), it is demanded that

$$
\begin{aligned}
\Delta H_{x}= & \frac{k d_{L}\left(\alpha_{2,3}+\alpha_{1,4}\right)}{-\varepsilon^{2}}+\frac{k\left(\alpha_{2,1}+\alpha_{1,6}\right)}{s} \\
& -\frac{\beta s\left(\alpha_{2,2} \rho_{2} \mu_{2}^{*}+\alpha_{1,5} \rho_{1} \mu_{1}^{*}\right)}{2 k^{2}}+O(\varepsilon) \rightarrow 0
\end{aligned}
$$

in which the first term is essential because in fact $\alpha_{2,3} \sim \alpha_{1,4} \sim O\left(\varepsilon^{2}\right)$. The exact higher order expressions for $\alpha_{2,3}$ and $\alpha_{1,4}$ are not given, since the order unity approximation found in (68) is sufficient to determine the flow field $(U, V, W)$ in the $\varepsilon \rightarrow 0$ limit.

Importantly, despite the difference between $\Delta H_{x}$ in two cases, (72) does not contradict (71), because the "effective pressure" condition (17b) for the strictly parallel field $(\varepsilon=0)$ case is otherwise satisfied in a stronger sense, that is, continuity of the tangential magnetic field, enabled by introducing extra eigenmodes in the oblique field case $(\varepsilon>0)$. Therefore, Eq. (68) obtained for $\varepsilon=0^{+}$that lives in a larger

(a)

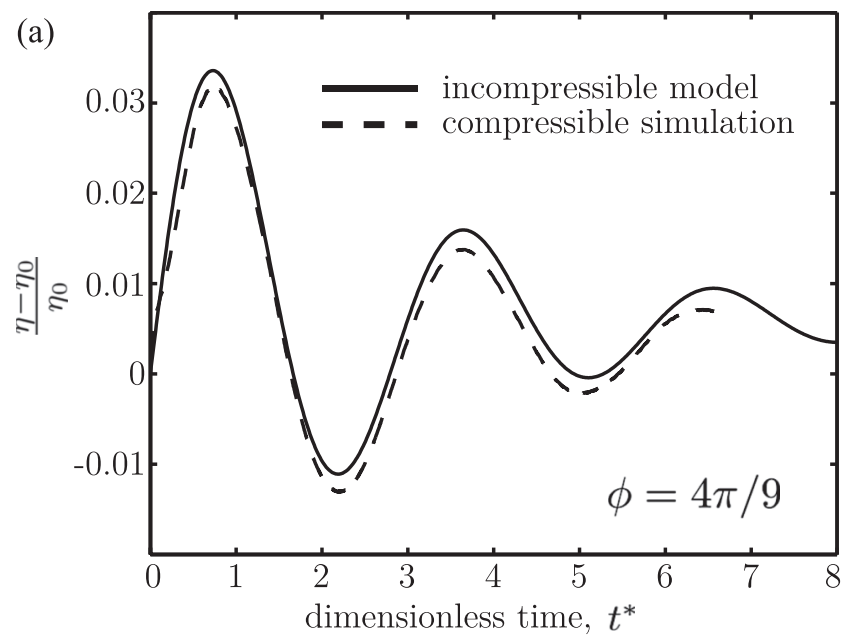

function space, as discussed in Sec. III B 2, provides an alternative solution. A detailed discussion on the linearization of original CD jump conditions (4) that give rise to the difference between Eq. (17) for $\varepsilon=0$ and (32) for $\varepsilon=0^{+}$, is given in Appendix D. The loss of continuity in the tangential magnetic field transitioning from $\varepsilon=0^{+}$to $\varepsilon=0$ is the result of non-uniform convergence associated with interchanging two asymptotics: the linearization limit $\eta_{0} \rightarrow 0$ and the large angle limit $\varepsilon \rightarrow 0$.

The discontinuous jump in flow fields observed between strictly and nearly parallel field cases can nonetheless be reduced by decreasing $d_{L}$. In the ideal MHD limit, substituting $d_{L}=0$ into (68) gives

$$
\alpha_{2,1}=\alpha_{1,6}=\frac{\left(\rho_{2}-\rho_{1}\right) \beta \eta_{0} k s}{4 k^{2}+\beta \rho_{1} s^{2}+\beta \rho_{2} s^{2}}, \quad \alpha_{2,2}=\alpha_{2,3}=\alpha_{1,4}=\alpha_{1,5}=0
$$

which are identical to those coefficients found in (20), exactly recovering the flow field subject to a parallel magnetic background. Therefore, the difference between interface velocities $W$ evaluated using $\varepsilon=0$ and $\varepsilon=0^{+}$disappears as $d_{L} \rightarrow 0$, even though the Rankine-Hugoniot (RH) conditions for the tangential magnetic field in these two cases are physically different for all $d_{L} \geq 0$. Particularly, it can be seen from Eqs. (16) and (24) that $\Delta H_{x}$ evaluated at $d_{L}=\varepsilon=0$ has a nonzero sinusoidal behavior.

The key comparisons made in this section between the $\varepsilon=0$ case and the $\eta_{0} \ll \varepsilon=0^{+}$case can be summarized as follows:

$$
\begin{aligned}
& \forall d_{L}>0,\left.\quad W_{1,2}(0)\right|_{\varepsilon=0^{+}}-\left.W_{1,2}(0)\right|_{\varepsilon=0} \neq 0, \\
& \lim _{d_{L} \rightarrow 0}\left[\left.W_{1,2}(0)\right|_{\varepsilon=0^{+}}-\left.W_{1,2}(0)\right|_{\varepsilon=0}\right]=0, \\
& \forall d_{L} \geq 0,\left.\quad \Delta H_{x}\right|_{\varepsilon=0^{+}}-\left.\Delta H_{x}\right|_{\varepsilon=0}=-\left.\Delta H_{x}\right|_{\varepsilon=0} \neq 0 .
\end{aligned}
$$

We also note that the solution characteristics in the transitioning parameters region where $\eta_{0} \sim \varepsilon \ll 1$ is unknown to the present linear analysis.

\section{NUMERICAL RESULTS}

The temporal behavior of the vector fields of interest is retrieved from the $s$-dependent solutions calculated in Sec. III, by applying the

FIG. 2. MHD interface perturbation amplitude histories. (a) Comparison of the incompressible model and the simulated $\eta$ histories corresponding to the shock driven case with $\phi=4 \pi / 9$. (b) Incompressible model $\eta$ histories for the same parameters with varying $\phi$.

(b)

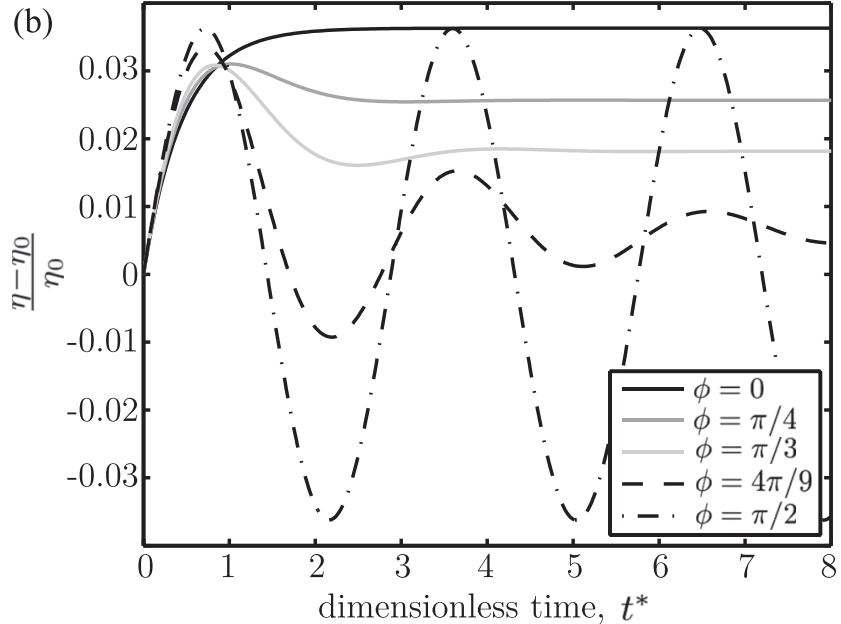




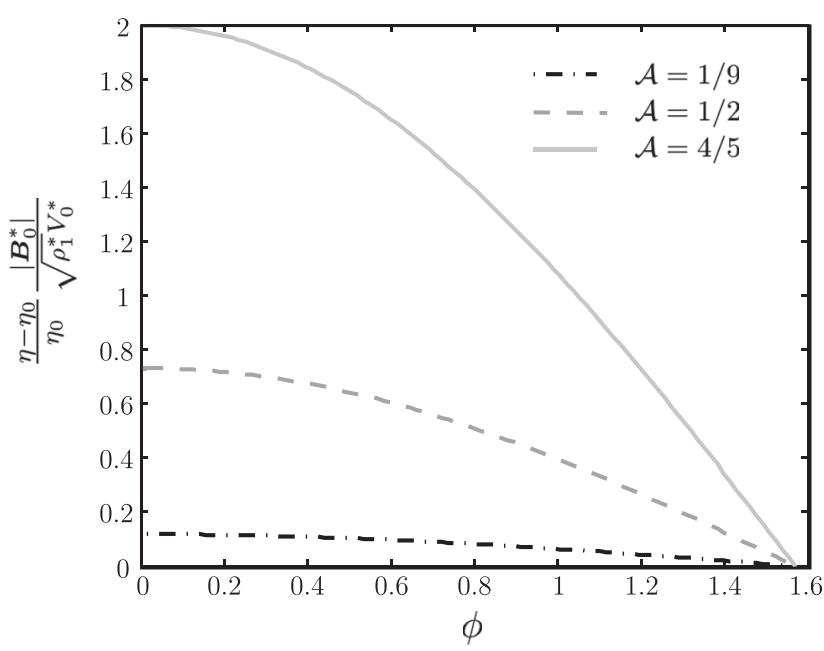

FIG. 3. MHD normalized limiting interface perturbation amplitude for varying $\phi$ and Atwood number.

inverse Laplace transform. For general parameter values of the Larmor radius $d_{L}$ and the plasma parameter $\beta$, this is performed numerically for a given time, $t$, using the multi-precision Gaver-Stehfest method. ${ }^{37-39}$ The algorithm originates from constructing a converging sequence of exponential probability density functions that naturally connects the expectation of a time-dependent function $\mathbb{E}[g(t)]$, to its Laplace transform $G(s)$. At a given time $t$, the temporal function value is thus approached by sampling its known transform at $2 M$ different real points, namely

$$
g_{M}(t) \equiv \frac{\ln 2}{t} \sum_{k=1}^{2 M} \psi_{k} G\left(\frac{k \ln 2}{t}\right) \stackrel{M \rightarrow \infty}{\longrightarrow} g(t)
$$

with $\psi_{k}$ being weights given by

$$
\psi_{k}=(-1)^{M+k} \sum_{j=\lfloor(k+1) / 2\rfloor}^{\min \{k, M\}} \frac{j^{M+1}}{M !}\left(\begin{array}{c}
M \\
j
\end{array}\right)\left(\begin{array}{c}
2 j \\
j
\end{array}\right)\left(\begin{array}{c}
j \\
k-j
\end{array}\right) .
$$

In a symbolic environment, such as MATHEMATICA ${ }^{\circledR}$, where the Gaver-Stehfest algorithm is implemented, the system precision is set at $5 M / 2$. Convergent results are obtained by gradually increasing $M$ up to $M=30$. Particularly, the numerical solutions for the limiting parameters match those given by the exact asymptotic expressions available in Sec. IV. It is also noted that since $\eta_{0}$ appears as a common factor of all quantities of interest, unity is assumed for its value in the subsequent numerical results, unless otherwise stated.

Additionally, a shock-driven compressible simulation for the RM flow that the present incompressible theory attempts to model is performed using the ideal MHD equations, in order to validate the analysis developed in Sec. IV A. For this simulation, a shock wave of Mach number $M$, traveling in the positive $z$-direction approaches the density interface as depicted in Fig. 1(c). A slightly different renormalization scheme is used for the compressible simulation. Here, dimensionless variables denoted by the asterisk are defined as

$$
\begin{aligned}
& \rho^{*}=\frac{\rho}{\rho_{0}}, \quad p^{*}=\frac{p}{p_{0}}, \quad t^{*}=\frac{t}{\Lambda / \sqrt{p_{0} / \rho_{0}}}, \\
& \boldsymbol{u}^{*}=\frac{\boldsymbol{u}}{\sqrt{p_{0} / \rho_{0}}}, \quad \boldsymbol{B}^{*}=\frac{\boldsymbol{B}}{\sqrt{\mu_{0} p_{0}}},
\end{aligned}
$$

where $\rho_{0}$ and $p_{0}$ are the initial equilibrium density and pressure upstream of the shock, respectively. Therefore, the simulation prescribes $\rho_{1}^{*}=1, \rho_{2}^{*}=1.25, p_{0}^{*}=1, \eta_{0}=0.01, \quad$ and $\quad \phi=4 \pi / 9$. Furthermore, the strengths of the driving shock and the applied magnetic field are characterized by $\bar{\beta}=2 \mu_{0} p_{0} / B_{0}^{2}=16$ and $M=U_{s} / a=1.1$, respectively, with $U_{s}$ being the shock speed and $a$ the upstream sound speed. As a result of the shock-interaction process with the interface, both fluids and consequently the interface perturbation are compressed on a timescale typically much shorter than that over which the RMI evolves. The magnetic field is also altered. Linear models such as the one described here most closely approximate the evolution of the post-shockcompression flow field. ${ }^{27}$ Thus, post-shock-compression parameter values are used as the initial conditions in the model, which are $\quad \rho_{1}^{*}=1.097, \rho_{2}^{*}=1.372, B_{0 x}^{*}=0.381, B_{0 z}^{*}=0.061, \quad$ and $\eta_{0}=0.00874$. The velocity imparted to the interface by the shock interaction process in the compressible problem is used as the impulse (a)

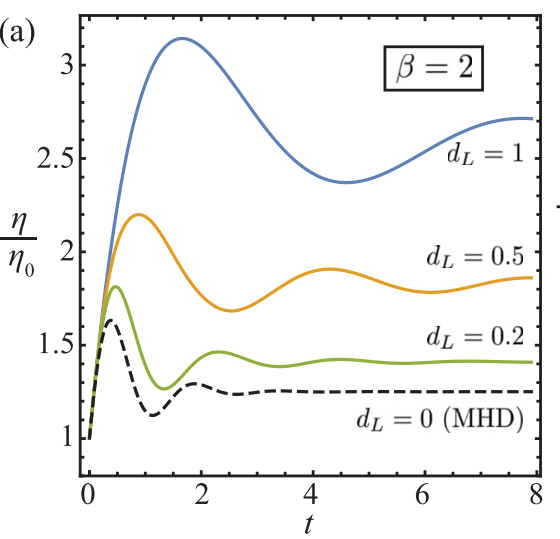

(b)

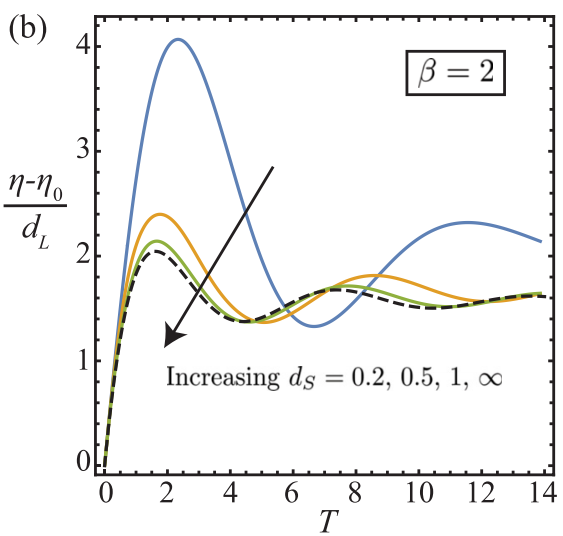

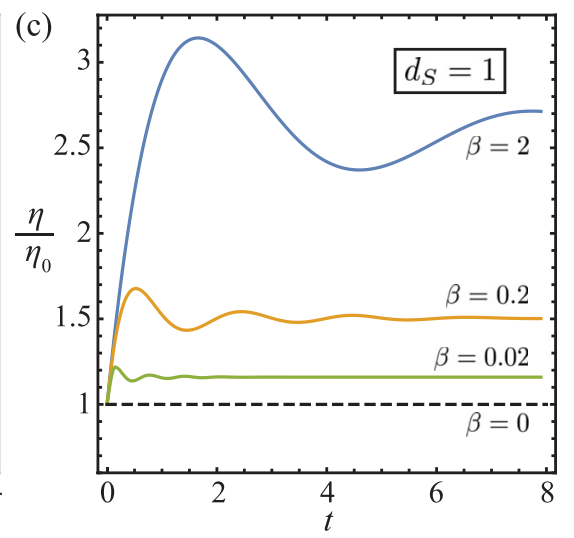

FIG. 4. The growth of the interfacial perturbation amplitude obtained at an oblique magnetic field angle $\phi=70^{\circ}$. Convergence toward (a) the MHD solution via decreasing $d_{L}$, (b) the large skin depth limit via increasing $d_{L}=d_{S}$ at a constant $\beta=2$, and (c) the strong magnetic field limit via decreasing $\beta$ at a constant $d_{S}=1$ is shown. The Atwood number $\mathcal{A}=0.5$ is held constant. 
(a)

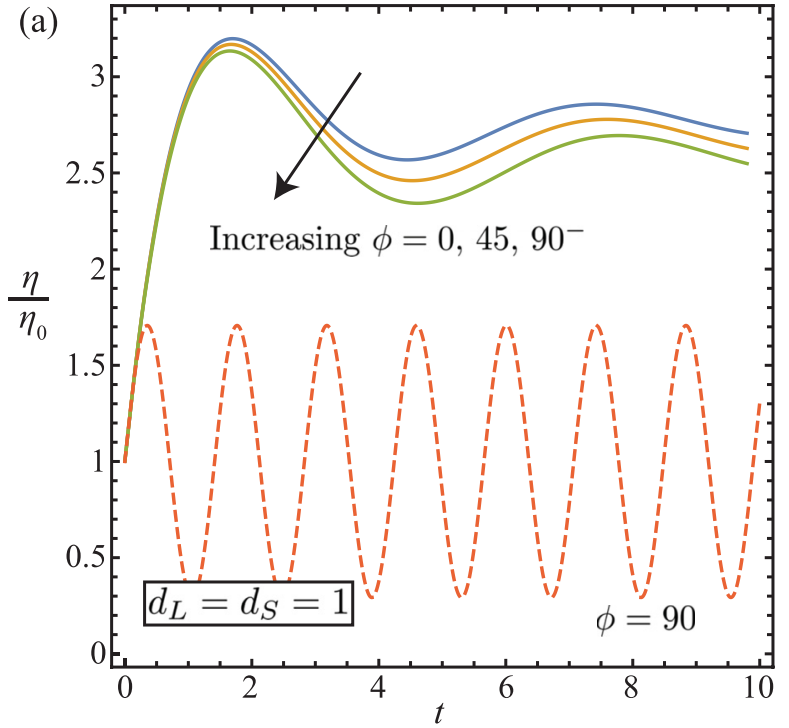

(b)

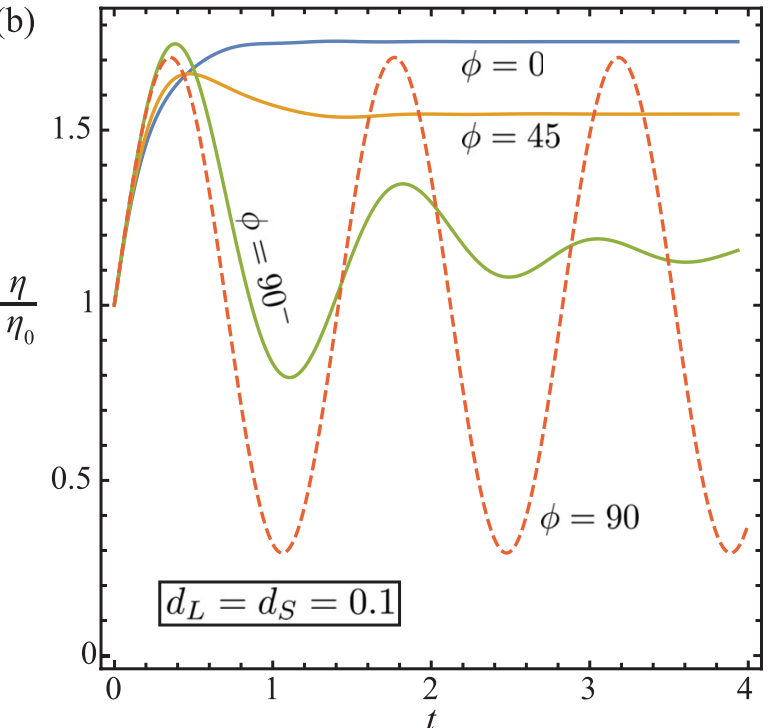

FIG. 5. Interfacial perturbation growth as a function of the magnetic field angle $\phi$. Explicit comparison between $0 \leq \phi<90^{\circ}$ and $\phi=90^{\circ}$ is made for $d_{L}=d_{S}=1$ in (a) and $d_{L}=d_{S}=0.1$ in (b). $\mathcal{A}=0.5$ is fixed in both cases.

magnitude in the model, which here is $V_{0}^{*}=0.113$. The shock-driven non-linear simulation subsequently compared to the model was carried out with the compressible ideal MHD code described in Ref. 27. It implements an eight-wave Riemann solver within an unsplit upwinding method. ${ }^{40}$ The divergence constraint on the magnetic field is enforced at each time step using a projection method. ${ }^{40}$ The simulation is twodimensional with boundary conditions periodic in the $x$-direction and zero-gradient at large $z$ in the $z$-direction. The simulation was conducted on a uniform grid with a cell size of $\Delta x=\Delta z=\Lambda / 1024$. This grid is sufficiently fine to predict the interface perturbation amplitude history to within $0.1 \%$ of the Richardson extrapolated exact value.

\section{A. Growth of the interface perturbation}

\section{The ideal MHD growth}

We first establish that under the ideal MHD description, the behavior of the interface in the oblique magnetic field case is a
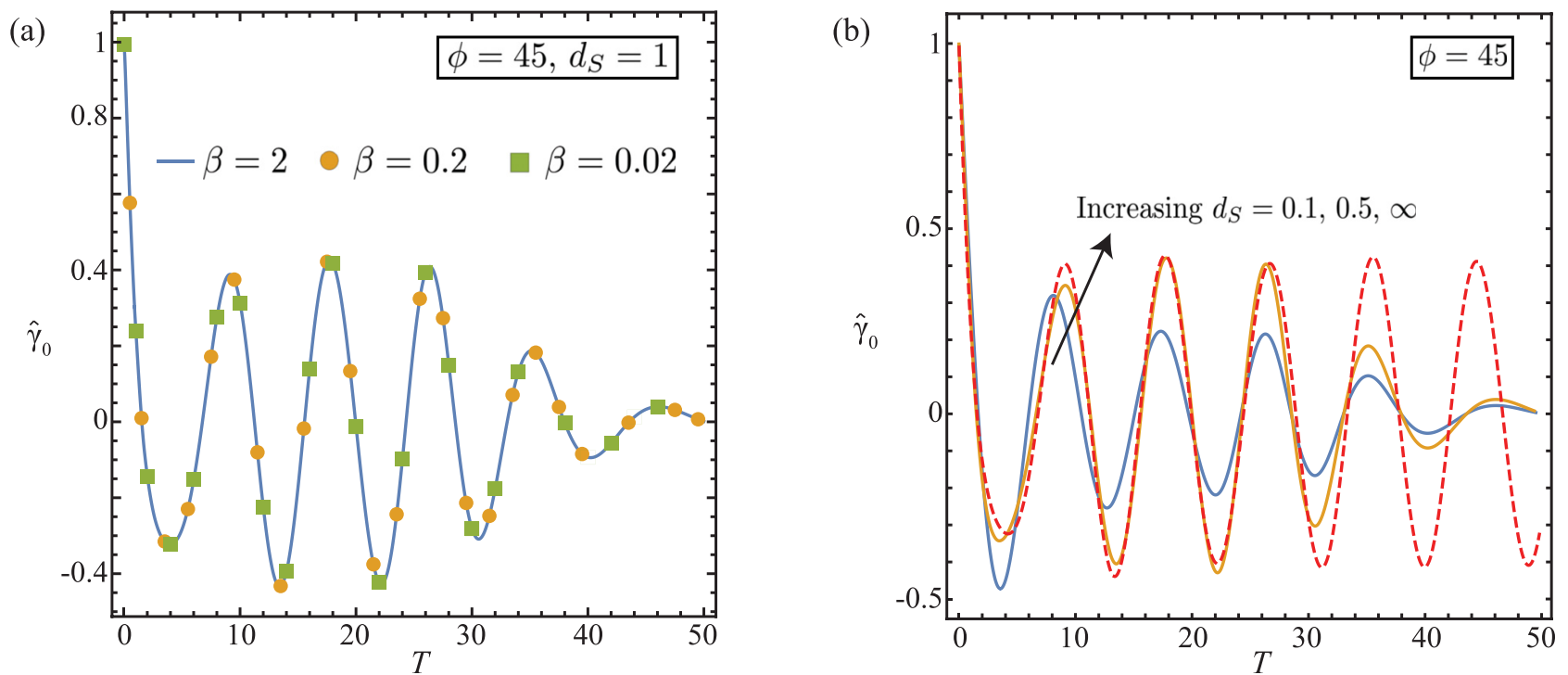

FIG. 6. Normalized circulation deposition $\hat{\gamma}_{0}$ at the interface in response to (a) decreasing $\beta=2,0.2,0.02$ and (b) increasing $d_{S}=0.1,0.5, \infty$. The magnetic field is imposed at an angle of $\phi=45^{\circ}$ and the cyclotron time $T$ is used for all series. 
(a)

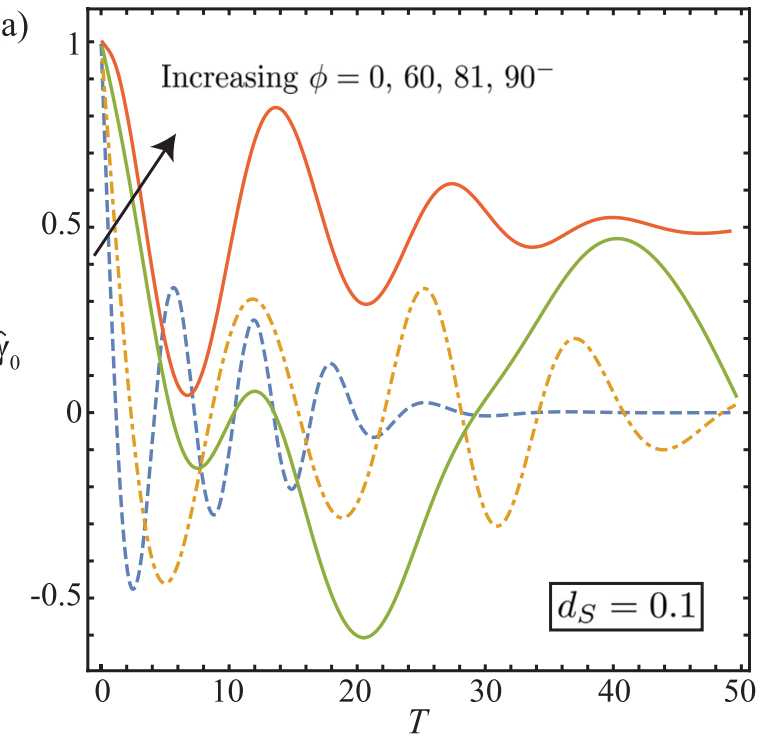

(b)

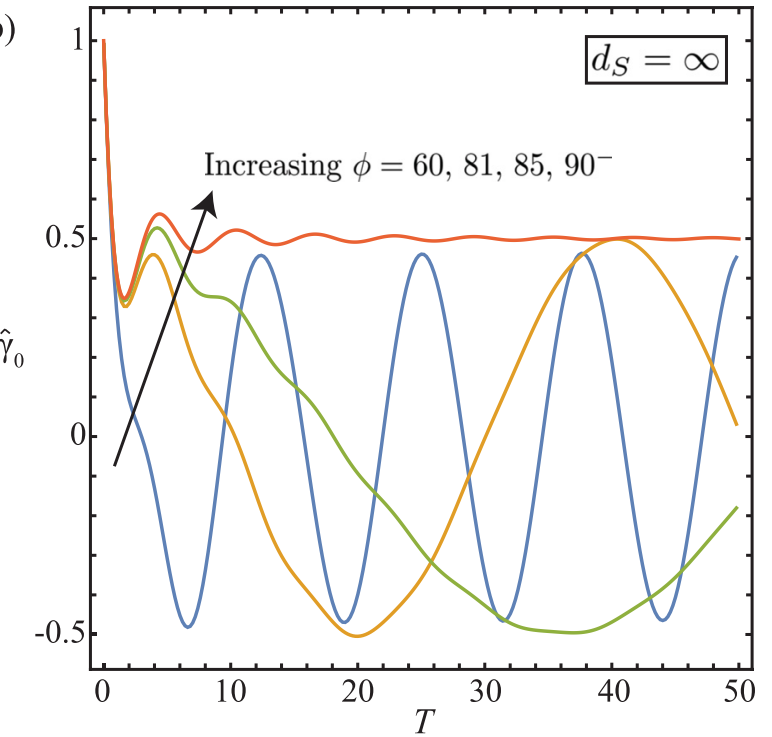

FIG. 7. Effect of the magnetic field angle $\phi$ on the circulation deposition $\hat{\gamma}_{0}$. In (a), $\phi$ is increased from 0 to the left limit of $90^{\circ}$ for finite $d_{S}=d_{L}=0.1$. In (b), the non-uniform convergence of $\hat{\gamma}_{0}$ as $\phi \rightarrow 90^{\circ}$ is highlighted for $d_{S}=\infty$. $\mathcal{A}=0.5$ is used throughout.
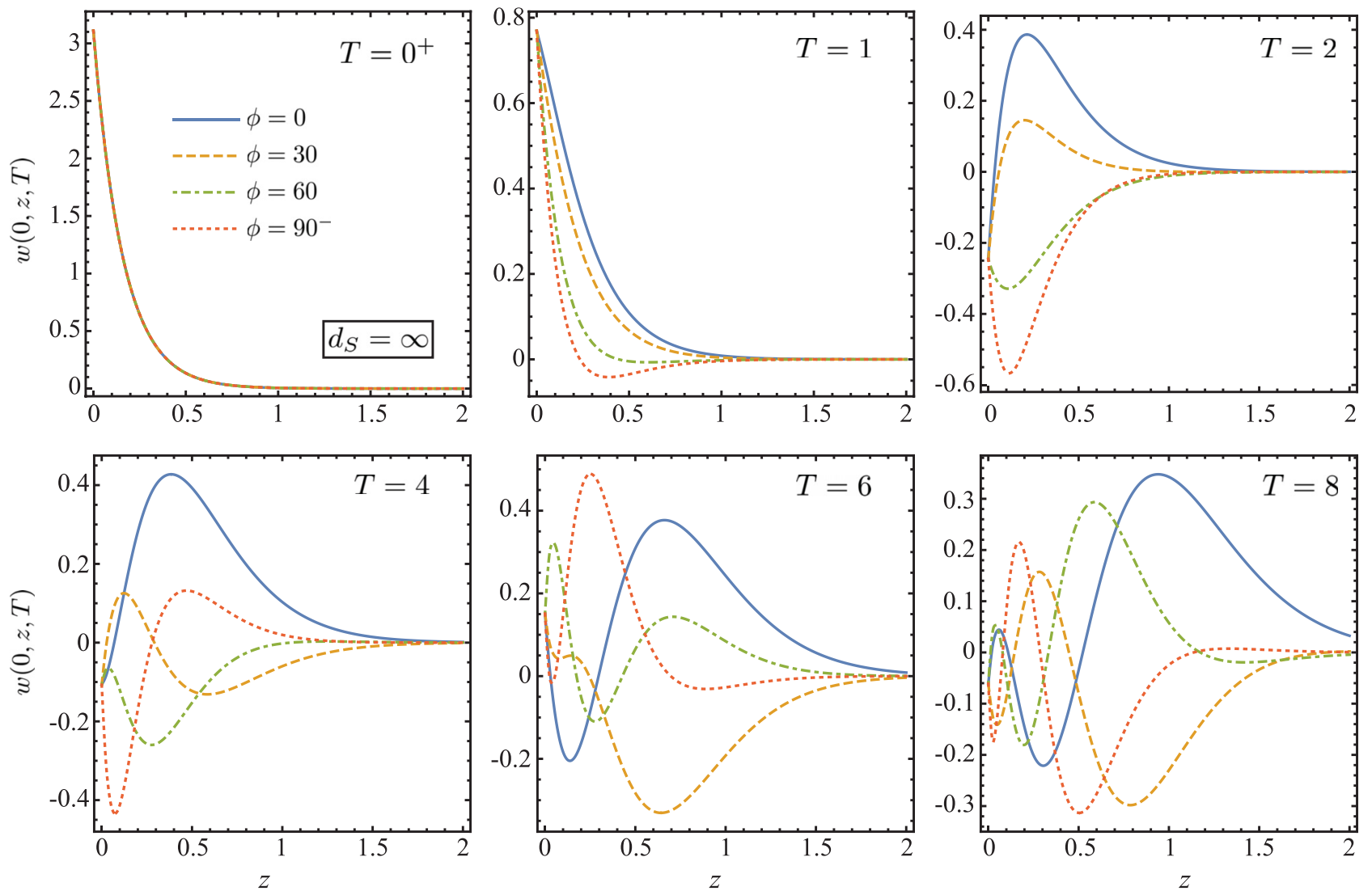

FIG. 8. Normal velocity profile $w(x=0, z, T)$ in the large $d_{S}$ limit. Six time instances from $T=0^{+}$, the initial impulse, to $T=8$ are taken for a range of magnetic field angles: $\phi=0$ (solid), $\phi=30^{\circ}$ (dashed), $\phi=60^{\circ}$ (dot-dashed), and $\phi \rightarrow 90^{\circ}$ (dotted). $\mathcal{A}=0.5$ in all cases. 
superposition of the oscillations of the transverse field case ${ }^{27}$ with the exponentially decaying growth rate of the normal field case. ${ }^{25}$ For the case shown in Fig. 2(a), where predictions made by the incompressible model (see Sec. IV A) are compared to the $\eta$ history extracted from the shock-driven compressible simulation, the incompressible model accurately predicts both the frequency and decay rate of the oscillations, while slightly overpredicting the amplitude, which also occurred for the models of the limiting cases. ${ }^{25,27}$

The interface behavior predicted by the model for a range of initial magnetic field angles (other parameters unchanged) is shown in Fig. 2(b). For $\phi \neq \pi / 2$, the interface perturbation amplitude, as $t^{*} \rightarrow \infty$, tends to a limit $\eta_{\infty}$ that is a function of $\phi$. Taking the large time limit of (35) yields the following closed form expression:

$$
\frac{\eta_{\infty}-\eta_{0}}{\eta_{0}}=\frac{V_{0}^{*} \sqrt{\rho_{1}^{*}}}{B_{0}^{*}}\left(\sqrt{\frac{\rho_{2}^{*}}{\rho_{1}^{*}}}-1\right) \cos \phi .
$$

For $\phi=0$, the limiting behavior of the normal field case ${ }^{25}$ is recovered. The general $\phi$ case exhibits the same linear dependence on $V_{0}^{*}$ and inverse dependence on the base field magnitude $B_{0}^{*}$. The scaling with $\cos \phi$ demonstrates that the long term suppression of the RMI is the strongest for fields that are nearly tangential to the interface. For $\phi=\pi / 2$, the interface oscillates in time without decay and the limiting amplitude does not exist. The dependence of $\eta_{\infty}$ on the problem parameters is illustrated in Fig. 3, where strong scaling of the asymptotic amplitude with the Atwood number is evident.

\section{Hall-MHD: Effect of finite Larmor radius}

We next explore the effect of finite Larmor radius $d_{L}$ and ion skin depth $d_{S}$, introduced in the Hall-MHD model, on the interface behavior. First, the RMI predicted by the linearized Hall-MHD equations is also inhibited in the presence of an oblique magnetic field. For instance, Fig. 4 shows the stabilizing evolution of the interface perturbation amplitude $\eta$ when the magnetic field is applied at an angle of $\phi=70^{\circ}$. A wide range of the parameter space is explored in this case. In (4a), the Larmor radius $d_{L}$ is decreased to show convergence toward the MHD result, measured in the original timescale $t$, through faster decaying oscillations and smaller perturbation growth upon saturation; in (4b), a similar pattern is observed for the rescaled amplitude $\eta / d_{L}$ in the cyclotron timescale $T$, when the large skin depth limit, $d_{S} \rightarrow \infty$, is approached with a fixed plasma energy ratio $\beta$. The fact that the interfacial oscillation frequency measured in $T$ now varies with different $d_{S}$ fundamentally differs from the normal field solution $(\phi=0)$ discussed by Shen et al., ${ }^{30}$ where the interfacial oscillation synchronizes exactly with the ion cyclotron frequency. The enhanced suppression effect for the RMI in response to a stronger background magnetic field strength is illustrated in (4c) where $\beta$ is decreased while holding $d_{S}$ constant, showing that $\eta$ decays inversely proportional to the external field strength, as discussed in Sec. IV C.
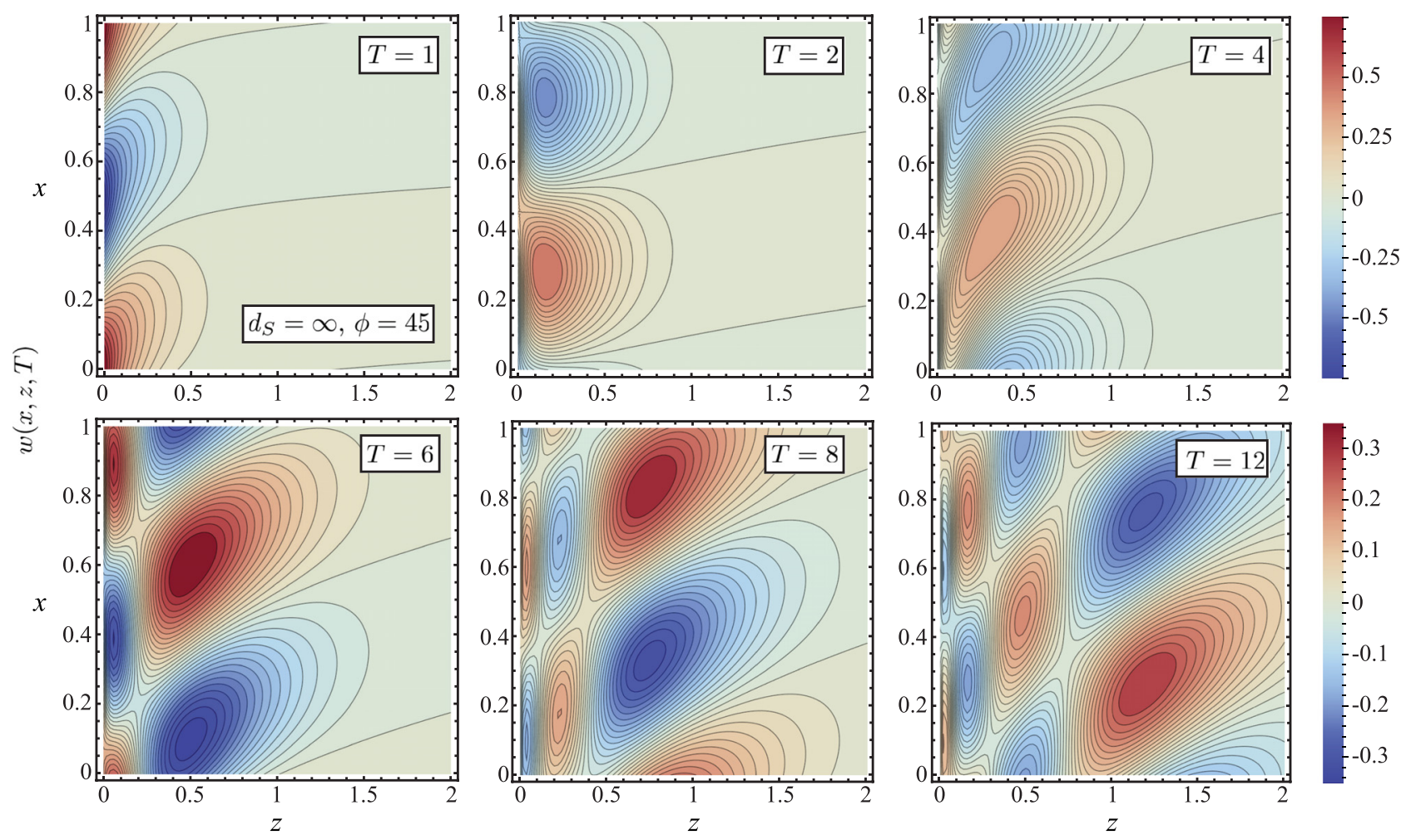

FIG. 9. Two-dimensional contours of the normal velocity field $w(x, z, T)$ in the large $d_{S}$ limit for an oblique magnetic field with $\phi=45^{\circ}$ and a plasma Atwood number $\mathcal{A}=0.5$. Two different color scales are assigned for $T=1,2,4$ and $T=6,8,12$, respectively. 
Next, we investigate the effect of changing $\phi$ in the Hall-MHD model in Fig. 5, where comparisons for the interface growth are made for the entire spectrum $0 \leq \phi \leq 90^{\circ}$ between two cases obtained for relatively large and small values of $d_{L}$ given in 5(a) and 5(b), respectively. In both cases, the singular jump between the asymptotic of $\phi \rightarrow 90^{\circ}$ and the exact solution of $\phi=90^{\circ}$ is observed (see Sec. IV D). The size of such a jump continuously decreases with decreasing $d_{L}$ : it is maximized in the large $d_{S}$ limit where the growth of $\eta$ collapses for all $\phi<90^{\circ}$, and eliminated in the MHD limit when $d_{L}=0$.

Furthermore, it is clear that the interfacial oscillation becomes a strong function of the magnetic field angle for a small $d_{L}$. In this region, a normal field $(\phi=0)$ excites oscillation due to the ion cyclotron motion, ${ }^{30}$ whereas a tangential field $\left(\phi=90^{\circ}\right)$ induces an oscillating phase of the flow field immediately away from the CD due to traveling waves along the mean interface. ${ }^{27}$ For a generally oblique field $\left(0<\phi<90^{\circ}\right)$, a combination of these two mechanisms results.

\section{B. Circulation deposition}

A distinct feature of the Hall-MHD model that contrasts the regular MHD theory is that its CD jump condition supports a tangential slip velocity across the interface that leads to a vortex sheet where circulation is deposited. We first demonstrate that the circulation deposition $\hat{\gamma}_{0}$, as a time series in $T$, is independent of $\beta$ and hence $d_{L}$, for any (a): Inital impulse, $t=0^{+}$

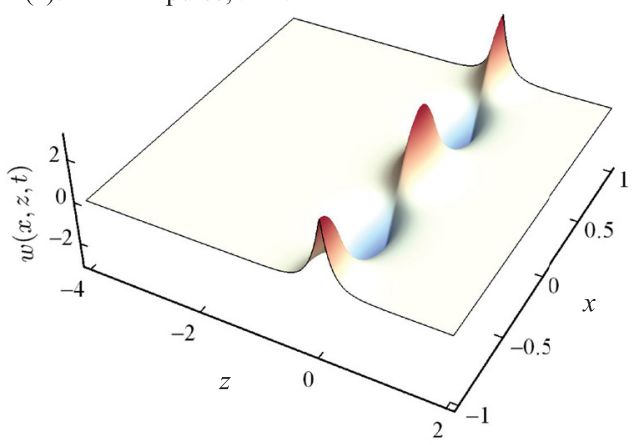

(b): MHD, $t=0.5$

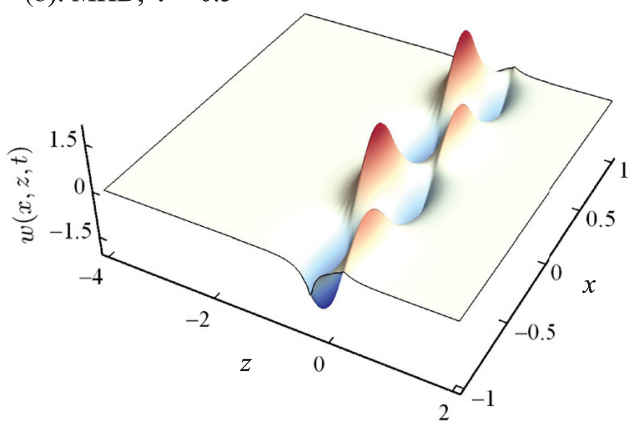

(c): MHD, $t=2$

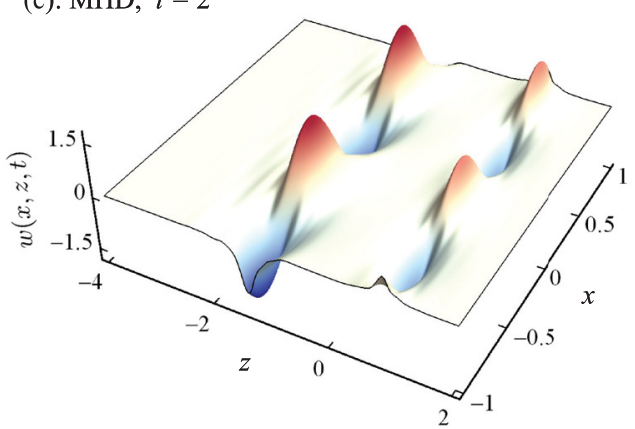

(d): Hall-MHD, $t=0.5$

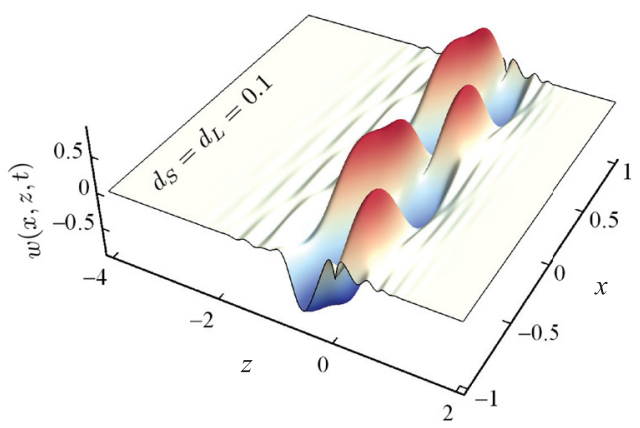

(e): Hall-MHD, $t=1$

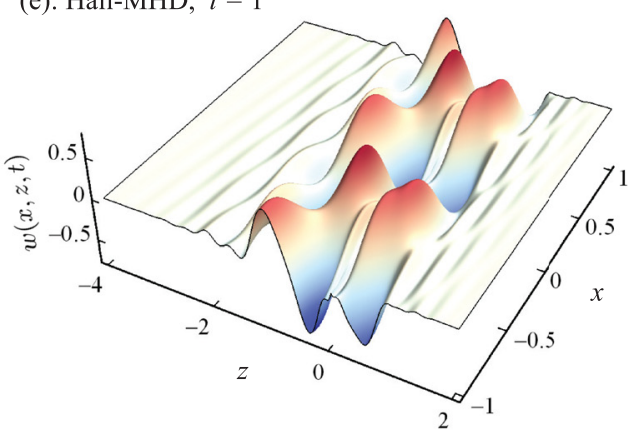

(f): Hall-MHD, $t=2$

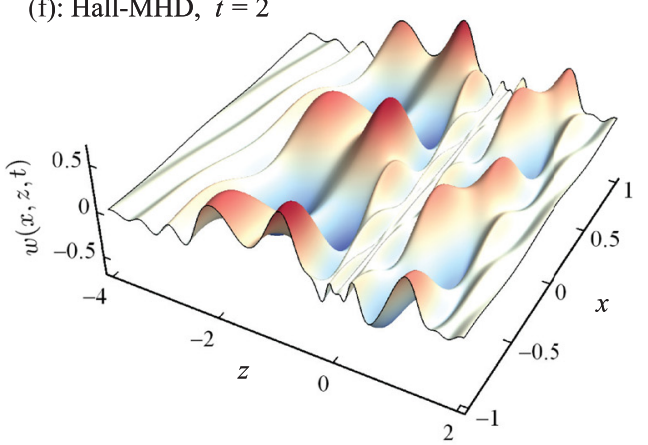

FIG. 10. Three-dimensional surfaces of the normal velocity field $w(x, z, t)$, as a function of time $t$, resulting from the MHD model [(b)-(c)] where $d_{L}=d_{S}=0$ and the HallMHD model $[(\mathrm{d})-(\mathrm{f})]$ where $d_{L}=d_{S}=0.1$. Both systems share a common initial impulse given in (a), as well as an Atwood number of $\mathcal{A}=0.5$. The results are shown over two wavelengths, $x \in[-1,1]$, to highlight the wave propagation along the magnetic field with $\phi=45^{\circ}$. 
given $0 \leq \phi<\pi / 2$. This can be verified by substituting $\beta=2 d_{L}^{2} / d_{S}^{2}$ into the Laplace transform of (36) to yield an expression of the form, $\Delta U / d_{L}=f\left(d_{S}, \phi ; \sigma\right)$, where the known function $f$ does not depend on $\beta$ nor $d_{L}$. For example, at $\phi=45^{\circ}$, Fig. 6(a) shows the collapse of $\hat{\gamma}_{0}$ as the time series in $T$, obtained for a decreasing $\beta$ while holding $d_{S}=1$ constant, to a universal curve that decays as $T \rightarrow \infty$. However, in the original time frame $t$, decreasing $\beta$ by increasing the imposed magnetic field strength in this case will rescale the collapsed curve into a smaller time window of $t$, giving faster oscillation and decay. This is because $T=t / d_{L}$ and reducing $\beta$ with a fixed $d_{S}$ simultaneously decreases $d_{L}$.

Therefore, it is convenient to focus on the collapsed series and study the effect of changing $d_{S}$ and $\phi$. The former is examined in Fig. 6(b), where $d_{S}$ is increased from 0.1 to 0.5 for $\phi=45^{\circ}$, giving rapid convergence toward the $d_{S}=\infty$ limit, while a slower decay of the oscillation amplitude is observed as $d_{S} \rightarrow \infty$. As an aside, such behavior is in general different from the results computed using the IICE model, ${ }^{30}$ where the decay rate of $\hat{\gamma}_{0}$ mildly rises when $d_{S}$ increases, due to the compressible flow treatment for the electrons. The effect of increasing $\phi$ is shown in Fig. 7. By covering the entire range of $0 \leq \phi<90$ in Fig. 7(a) for constant $d_{S}=0.1$, it is established that following a transitioning period during which the behavior of $\hat{\gamma}_{0}$ converges to the limiting solution given by the $\phi \rightarrow 90^{\circ}$ asymptotic, a consistently decaying oscillation whose amplitude and period increase with $\phi$ is always reached. The convergence as $\phi \rightarrow 90^{\circ}$ is non-uniform in T and emphasized in Fig. 7(b) where $d_{S}=\infty$ is chosen to exaggerate the transitioning period. In this case, although it can be shown from Eqs. (36) and (57) that $\left.\hat{\gamma}_{0}(T)\right|_{\phi=\pi / 2} \rightarrow 1 / 2$ as $T \rightarrow \infty$, the actual time series corresponding to $\phi<\pi / 2$ must deviate away from the limiting solution and undertake smooth oscillations around zero for a sufficiently large $T$.

Additionally, the discontinuous jump in $\hat{\gamma}_{0}$ between the $\phi \rightarrow \pi / 2$ solution given in Fig. 7 , and the $\phi=\pi / 2, d_{L}$-independent solution becomes clear after recalling from Eqs. (24) and (36) that for all $d_{L} \geq 0$

$$
\left.\hat{\gamma}_{0}(t)\right|_{\phi=\pi / 2}=\cos \left(\frac{4 \pi t}{\sqrt{\beta\left(\rho_{1}+\rho_{2}\right)}}\right) .
$$

Indeed, the simple cosine wave given by (79) for $\phi=\pi / 2$ differs from the limiting solution as $\phi \rightarrow \pi / 2$, plotted using Eq. (60), again validating the analysis of Sec. IV D.

\section{Normal velocity profile}

A curious observation made in Sec. IV B 1 concerns the cause of an universal interface growth that is independent of the magnetic field angle $\phi$ in the large $d_{S}$ limit, even though the circulation deposition in this limit does vary with $\phi$ [see Fig. 7(b)]. To demonstrate, Fig. 8 depicts the evolution of the normal velocity profile $w^{\prime}$ in time $T$ from the initial impulse, across the line $\{(x, z) \mid x=0, z \in[0,2]\}$, for a range of $\phi$. The results are drawn for $z>0$ since $w^{\prime}$ is an even function of $z$ when $d_{S}=\infty$. It is clear that the flow profile away from the interface reduces its propagation speed in the normal direction as the field angle increases, while preserving a common velocity at the interface $z=0$ for all time $T$.

Figure 9 further explores the propagation characteristics as density plots for $w^{\prime}$ over the two-dimensional domain $\{(x, z) \mid x$ $\in[0,1], z \in[0,2]\}$, noting that the flow profile is periodic in $x$ and symmetric about the $x$-axis. At a particular angle $\phi=45^{\circ}$, it is seen that while deforming, the velocity contours travel in a direction that is aligned with the imposed magnetic field. Flow disturbances due to vorticity generation are constantly being produced and transported away from the $\mathrm{CD}$ due to the interface-normal component of the wave velocity, decreasing their influence on the interface dynamics. Meanwhile, along the CD at $z=0, w^{\prime}$ continuously changes its phase due to the interface-parallel component of the wave velocity and the oscillatory nature of the vorticity generation, necessarily causing oscillation of the interface. The net result of these two wave components provides the suppression mechanism for the RMI in the present Hall-MHD framework.

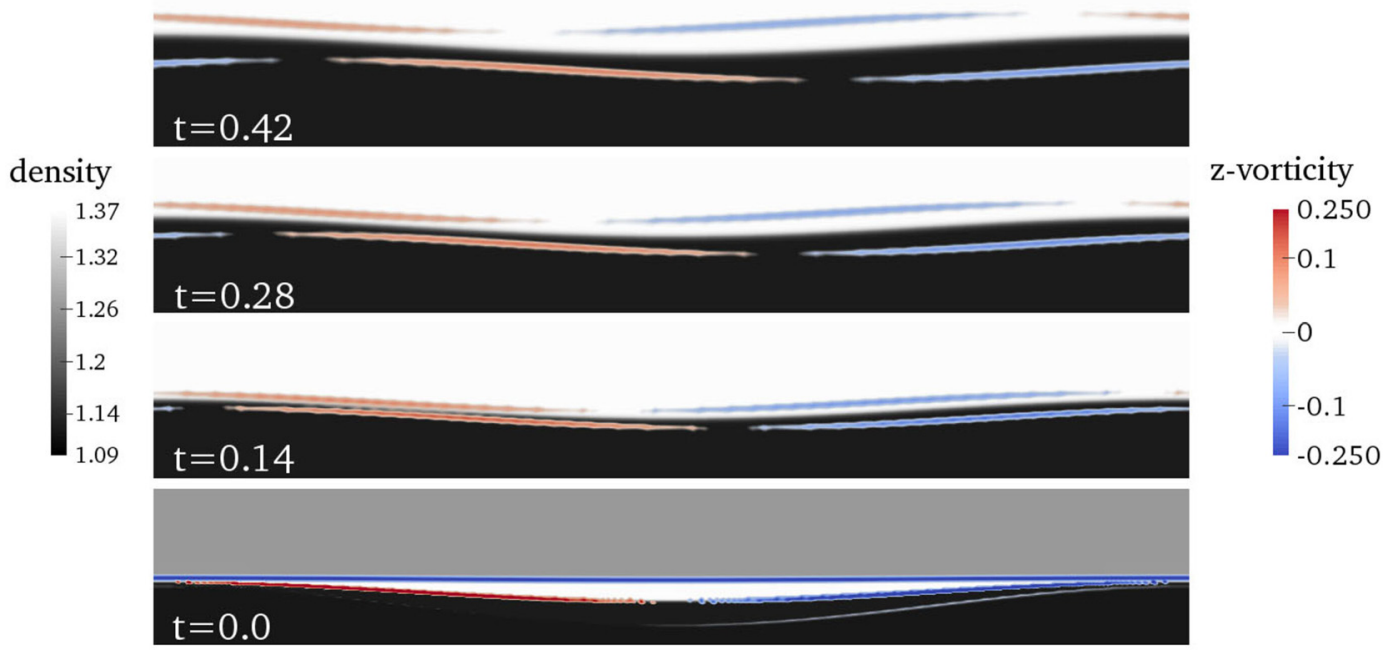

FIG. 11. Short term evolution of the post-shock vorticity field near the interface from the compressible simulation with $\phi=4 \pi / 9$. Frame timestamps are non-dimensionalized by $\Lambda \sqrt{\rho_{1}^{*} / p_{0}^{*}}$. 
The effect of finite ion skin depth and Larmor radius on the normal velocity distribution is examined in Fig. 10, where comparison is also made against the regular MHD theory. When the background magnetic field is applied at an oblique angle, e.g., $\phi=45^{\circ}$, the MHD solution exhibits a soliton-like behavior of the Alfvén waves in the magnetic field direction that originates from splitting the initial impulse into two fluids on each side of the interface. In contrast, a dispersive wave system available in the Hall-MHD model, including the ion cyclotron, the Alfvén and Whistler waves of finite frequencies, ${ }^{31}$ evidently complicates the flow propagation by introducing highly oscillatory patterns. This dispersive behavior in Hall-MHD develops from mild perturbation to the MHD solution immediately after the initial impulse, and gradually evolves into a long-time dominant feature of the flow field.

\section{Vorticity dynamics}

\section{Ideal MHD}

The interface dynamics in the ideal MHD case can be explained by examining the transport of vorticity in the presence of an oblique field. This is illustrated in Fig. 11, which shows the vorticity field overlaid on the density field at a series of times following the shock interaction in the nonlinear simulation. After the transmitted and reflected shocks depart the vicinity of the interface, this closely matches the vorticity transport predicted by the present model. As previously noted in Ref. 35, the circulation baroclinically generated at the interface by the shock interaction process is identical to the hydrodynamic case. Thus immediately following the shock interaction, before vorticity transport has had an opportunity to occur, the vorticity distribution induces the same initial growth rate as in the hydrodynamic case. Since the oblique magnetic field penetrates the interface, however, vorticity is forbidden from remaining on the interface by the ideal MHD Rankine-Hugoniot relations. In Fig. 11, it can be seen that this situation is resolved by the formation of waves traveling parallel and anti-parallel to the base magnetic field that bifurcate vorticity distribution and transport it from the interface. The interface parallel component of the vorticity transport continuously alters the phase of the normal velocities induced at the interface, which causes the interface perturbation growth rate to oscillate in time. The interface normal component of vorticity transport simultaneously causes the growth rate to decay as the vorticity distribution becomes more distant from the interface and hence the induced velocities there decrease.

The interface behavior in the $\phi \rightarrow 0$ and $\phi \rightarrow \pi / 2$ limits of the oblique field case coincides with earlier results for normal ${ }^{25}$ and tangential $^{27}$ fields. The earlier tangential field model, however, did not
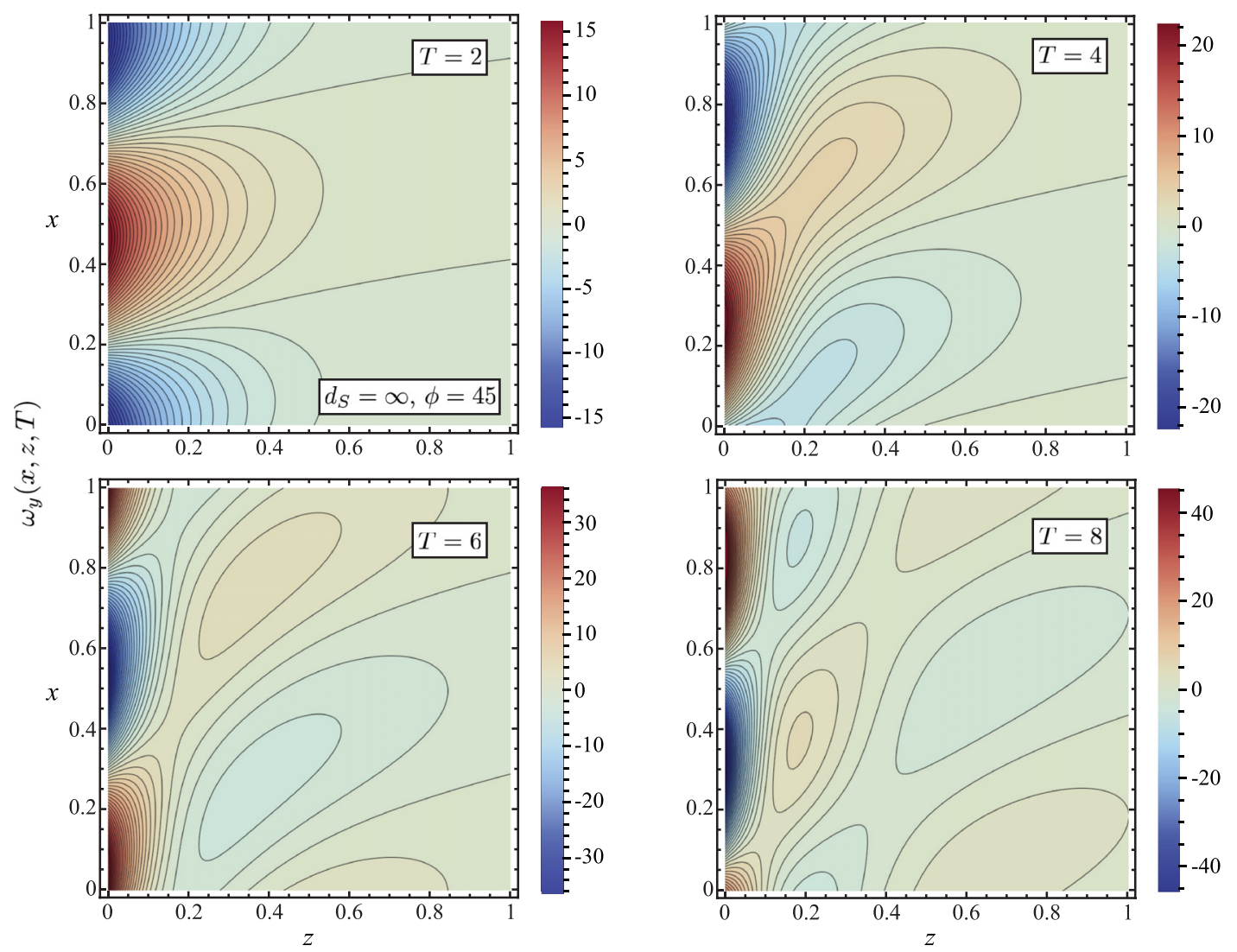

FIG. 12. Two-dimensional contours of the out-of-plane vorticity $\omega_{y}(x, z, T)$ in the $d_{S}=\infty$ limit for an oblique magnetic field with $\phi=45^{\circ}$. The color scale is reset for each time instance $(T=2,4,6,8)$ to show vorticity production as the flow evolves. 
resolve the vorticity carrying waves and instead integrated across them. The full structure of the flow in this case is revealed by the $\phi \rightarrow$ $\pi / 2$ limit of the oblique field model.

\section{Hall-MHD}

Different from the ideal MHD prediction, the production and propagation of the out-of-plane vorticity, $\omega_{y}$, in the Hall-MHD model is illustrated in Fig. 12 for the large $d_{S}$ limit, where the important features discussed in Sec. III C are well captured. As the flow evolves, a thin layer of flow in a neighborhood of the density interface at $z=0$ sees substantial vorticity injection due to the magnetic field perturbation, causing the growth of total circulation. This should not be confused with the circulation deposition at the interface due to shearing, which oscillates with a falling envelop, as shown in Sec. VB. The amount of vorticity production however decays rapidly away from the interface as $z$ increases, and the transport mechanism enabled by the imposed magnetic field thereby manifests. That is, the Alfvén waves traveling along the magnetic field are responsible for carrying away the vorticity at an oblique angle.

\section{CONCLUSIONS}

An incompressible model for the two-dimensional Hall-MHD RMI has been developed for an arbitrary initial magnetic field angle. The reduced growth of instability is established for all field orientations other than strictly out-of-plane. However, the suppression is most effective for near tangential fields but becomes less effective with increasing plasma length scales, namely the Larmor radius $d_{L}$ and the ion skin depth $d_{S}$.

The behavior of the interface was shown to be governed by the production and transport of vorticity via waves traveling along the magnetic field. In the ideal MHD limit, obtained by taking the ion skin depth to zero, the component of vorticity transport normal to the interface causes the perturbation growth rate to decay, while the component of transport parallel to the perturbation direction causes a continuous change in the phase of the induced velocities at the interface, resulting in growth rate oscillation. The interface behavior predicted in this case compares well with the results of nonlinear MHD simulations. For Hall-MHD flow of finite $d_{L}$ and $d_{S}$, oscillations associated with the ion cyclotron effect are imposed when the initial magnetic field is not strictly parallel to the interface. The normal component of the field in Hall-MHD allows vorticity production across the flow domain and introduces a dispersive wave system transporting the vorticity.

Other than the ideal MHD theory, asymptotic behavior of the general incompressible Hall-MHD RM flow is also studied for limiting values of the ion skin depth, the plasma kinetic-to-magnetic energy ratio, and the initial magnetic field angle. Analytical results are derived in each limit. When the appropriate limits for the initial field angle are taken, the present analysis replicates the predictions made by existing models for the normal and parallel field cases in both Hall-MHD and ideal MHD.

\section{ACKNOWLEDGMENTS}

This work was supported by the KAUST Office of Sponsored Research under Award No. URF/1/3418-01. Dr. Wheatley was supported by the Australian Research Council Discovery Early
Career Researcher Award (Project No. DE120102942) and the Australian Research Council's Discovery Projects funding scheme (Project No. DP120102378).

\section{APPENDIX A: EIGENVALUE SYSTEM}

In this section, we tabulate explicitly the matrix $A_{i}$ in the eigenvalue system (25) in Table I, and its eigenvectors $\boldsymbol{v}_{i, j}$ in Table II, as needed in (27) for solution construction.

TABLE I. Nonzero elements of matrix $A_{i}$ specified for Eq. (25).

$$
\begin{aligned}
& a_{1,2}=1 \\
& a_{2,1}=\frac{2 k^{2} B_{0 x}^{2}+\beta \rho_{i} s^{2}}{2 d_{L}^{2} s^{2}+2 B_{0 z}^{2}} \\
& a_{2,2}=-\frac{i k B_{0 x}\left(2 B_{0 z}^{2}+s^{2} d_{L}^{2}\right)}{s^{2} B_{0 z} d_{L}^{2}+B_{0 z}^{3}} \\
& a_{2,3}=\frac{\beta \rho_{i} s^{3} d_{L}}{2\left(s^{2} B_{0 z} d_{L}^{2}+B_{0 z}^{3}\right)} \\
& a_{2,5}=-\frac{i \beta k s^{2} B_{0 x} d_{L} \rho_{i}}{2\left(s^{2} B_{0 z} d_{L}^{2}+B_{0 z}^{3}\right)} \\
& a_{2,6}=-\frac{i \beta k s^{2} d_{L} \rho_{i}}{2\left(B_{0 z}^{2}+s^{2} d_{L}^{2}\right)} \\
& a_{3,4}=1 \\
& a_{4,1}=-\frac{d_{L}\left(2 B_{0 x}^{2}\left(2 k^{4}\left(B_{0 z}^{2}+s^{2} d_{L}^{2}\right)+\beta k^{2} s^{2} \rho_{i}\right)+\beta^{2} s^{4} \rho_{i}^{2}\right)}{2 \beta s B_{0 z} \rho_{i}\left(B_{0 z}^{2}+s^{2} d_{L}^{2}\right)} \\
& a_{4,2}=\frac{i k B_{0 x} d_{L}}{s}\left(\frac{s^{2}}{B_{0 z}^{2}+s^{2} d_{L}^{2}}+\frac{2 k^{2}}{\beta \rho_{i}}\right) \\
& a_{4,3}=\frac{\beta s^{2} \rho_{i}}{2\left(B_{0 z}^{2}+s^{2} d_{L}^{2}\right)}+k^{2} \\
& a_{4,4}=-\frac{i k B_{0 x}}{B_{0 z}} \\
& a_{4,5}=\frac{i k B_{0 x}}{2 s}\left(2 k^{2}-\frac{\beta s^{2} \rho_{i}}{B_{0 z}^{2}+s^{2} d_{L}^{2}}\right) \\
& a_{4,6}=-\frac{i \beta k s B_{0 z} \rho_{i}}{2\left(B_{0 z}^{2}+s^{2} d_{L}^{2}\right)}-\frac{i k^{3} B_{0 x}^{2}}{s B_{0 z}} \\
& a_{5,1}=-\frac{d_{L}\left(2 k^{2} B_{0 x}^{2}+\beta s^{2} \rho_{i}\right)}{\beta \rho_{i}\left(B_{0 z}^{2}+s^{2} d_{L}^{2}\right)} \\
& a_{5,2}=\frac{2 i k B_{0 x} B_{0 z} d_{L}}{\beta B_{0 z}^{2} \rho_{i}+\beta s^{2} d_{L}^{2} \rho_{i}} \\
& a_{5,3}=\frac{s B_{0 z}}{B_{0 z}^{2}+s^{2} d_{L}^{2}} \\
& a_{5,5}=-\frac{i k B_{0 x} B_{0 z}}{B_{0 z}^{2}+s^{2} d_{L}^{2}} \\
& a_{5,6}=\frac{i k s^{2} d_{L}^{2}}{B_{0 z}^{2}+s^{2} d_{L}^{2}} \\
& a_{6,5}=-i k
\end{aligned}
$$


TABLE II. Components of the eigenvector $v$ corresponding to a given eigenvalue $\lambda$, as seen in Eq. (27). The matrix entries $a_{i, j}$ are specified in Table I.

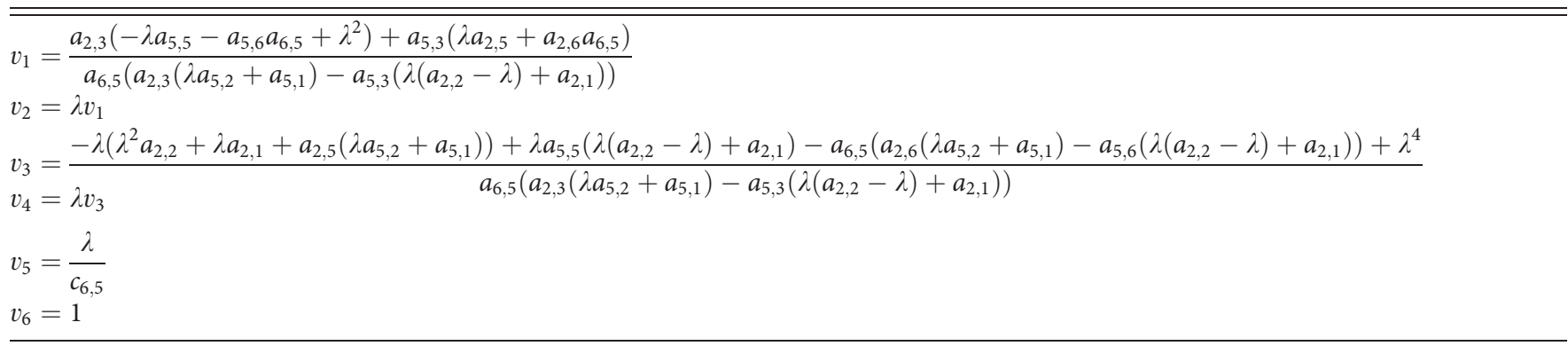

\section{APPENDIX B: LIMITING LINEAR EQUATIONS FOR COEFFICIENTS $\alpha$}

Here, we provide the linear systems derived in Sec. IV that are used to determine the coefficients $\alpha_{i, j}$ under various limits.

1. Large skin depth $\left(\beta \rightarrow 0, d_{L}<\infty\right)$

In Sec. IV B 1, the linear equations that lead to solution (55) have the following matrix representation:

$$
\left[\begin{array}{cccccc}
-1 & -1 & -1 & 1 & 1 & 1 \\
\frac{k \exp (-i \phi)}{s} & 0 & \frac{k d_{L}}{\cos \phi} & \frac{k d_{L}}{\cos \phi} & 0 & \frac{k \exp (i \phi)}{s} \\
\frac{k(\sin \phi+i \cos \phi)}{-s} & 0 & \frac{k d_{L} \sin \phi}{\cos ^{2} \phi} & \frac{k d_{L} \sin \phi}{\cos ^{2} \phi} & 0 & \frac{i k \exp (i \phi)}{s} \\
0 & 0 & 0 & C_{44} & -\frac{i \beta \rho_{1}}{2 k d_{L}} & 0 \\
0 & -\frac{i \beta \rho_{2}}{2 k d_{L}} & C_{53} & 0 & 0 & 0 \\
\frac{\rho_{2} s}{k} & \frac{\rho_{2} \sqrt{\sigma^{2}+1}}{k d_{L}} & C_{63} & C_{64} & \frac{\rho_{1} \sqrt{\sigma^{2}+1}}{k d_{L}} & \frac{\rho_{1} s}{k}
\end{array}\right]\left[\begin{array}{c}
\alpha_{2,1} \\
\alpha_{2,2} \\
\alpha_{2,3} \\
\alpha_{1,4} \\
\alpha_{1,5} \\
\alpha_{1,6}
\end{array}\right]=\left[\begin{array}{c}
0 \\
0 \\
0 \\
0 \\
0 \\
b
\end{array}\right],
$$

where

$$
\begin{array}{rlrl}
C_{44} & =\frac{\beta \rho_{1}\left(-2 s d_{L} \tan \phi+i\right)}{2 k d_{L}}+i k d_{L} \sec ^{2} \phi, & C_{53}=\frac{\beta \rho_{2}\left(2 s d_{L} \tan \phi+i\right)}{2 k d_{L}}+i k d_{L} \sec ^{2} \phi \\
C_{63} & =\frac{2 k d_{L} \sec ^{2} \phi}{\beta}+\frac{\rho_{2}\left(2-3 i s d_{L} \tan \phi\right)}{k d_{L}}, & C_{64}=\frac{2 k d_{L} \sec ^{2} \phi}{\beta}+\frac{\rho_{1}\left(2+3 i s d_{L} \tan \phi\right)}{k d_{L}} \\
b & =\eta_{0}\left(\rho_{2}-\rho_{1}\right) .
\end{array}
$$

In order to obtain the correct limiting solution for $\alpha_{i, j}$, it is sufficient to truncate the matrix entries at $O\left(\beta^{0}\right)$ in their Taylor expansions, except for those in the fourth and fifth row, where truncation is made at $O(\beta)$.

2. Large skin depth $\left(d_{L} \rightarrow \infty, \beta>0\right)$

Similarly, the following limiting linear system gives rise to solution (63) in Sec. IV B 2:

$$
\left[\begin{array}{cccccc}
-1 & -1 & -1 & 1 & 1 & 1 \\
\frac{k \exp (-i \phi)}{\sigma \epsilon} & 0 & \frac{k \sec \phi}{\epsilon} & \frac{k \sec \phi}{\epsilon} & 0 & \frac{k \exp (i \phi)}{\sigma \epsilon} \\
\frac{k(\sin \phi+i \cos \phi)}{-\sigma \epsilon} & 0 & \frac{k \tan \phi \sec \phi}{\epsilon} & \frac{k \tan \phi \sec \phi}{\epsilon} & 0 & \frac{i k \exp (i \phi)}{\sigma \epsilon} \\
0 & 0 & 0 & C_{44} & -\frac{i \beta \rho_{1} \epsilon}{2 k} & 0 \\
0 & -\frac{i \beta \rho_{2} \epsilon}{2 k} & C_{53} & 0 & 0 & 0 \\
\frac{\rho_{2} \sigma \epsilon}{k} & \frac{\rho_{2} \sqrt{\sigma^{2}+1} \epsilon}{k} & C_{63} & C_{64} & \frac{\rho_{1} \sqrt{\sigma^{2}+1} \epsilon}{k} & \frac{\rho_{1} \sigma \epsilon}{k}
\end{array}\right]\left[\begin{array}{c}
\alpha_{2,1} \\
\alpha_{2,2} \\
\alpha_{2,3} \\
\alpha_{1,4} \\
\alpha_{1,5} \\
\alpha_{1,6}
\end{array}\right]=\left[\begin{array}{c}
0 \\
0 \\
0 \\
0 \\
0 \\
b
\end{array}\right],
$$

where 


$$
\begin{aligned}
& C_{44}=\frac{\beta \rho_{1} \epsilon(-2 \sigma \tan \phi+i)}{2 k}+\frac{i k \sec ^{2} \phi}{\epsilon}, \quad C_{53}=\frac{\beta \rho_{2} \epsilon(2 \sigma \tan \phi+i)}{2 k}+\frac{i k \sec ^{2} \phi}{\epsilon}, \\
& C_{63}=\frac{2 k \sec ^{2} \phi}{\beta \epsilon}+\frac{\rho_{2} \epsilon(2-3 i \sigma \tan \phi)}{k}, \quad C_{64}=\frac{2 k \sec ^{2} \phi}{\beta \epsilon}+\frac{\rho_{1} \epsilon(2+3 i \sigma \tan \phi)}{k} .
\end{aligned}
$$

Here, the entries are truncated at $O\left(\epsilon^{0}\right)$ for the first three rows and at $O(\epsilon)$ for the last three rows.

\section{Large angle limit}

Again, in the limiting case of $\phi \rightarrow \pi / 2$, or equivalently, $\varepsilon \rightarrow 0$ studied in Sec. IV D, the flow coefficients are determined from

$$
\left[\begin{array}{cccccc}
-1 & -1 & -1 & 1 & 1 & 1 \\
-\frac{i k}{s} & \frac{i s \beta \rho_{2}}{2 k} & \frac{k d_{L}}{\varepsilon}-\frac{i s \beta \rho_{2}}{2 k} & \frac{k d_{L}}{\varepsilon}+\frac{i s \beta \rho_{1}}{2 k} & -\frac{i s \beta \rho_{1}}{2 k} & \frac{i k}{s} \\
-\frac{k}{s} & \frac{s \beta \mu_{2}^{*} \rho_{2}}{2 k^{2}} & C_{33} & C_{34} & \frac{s \beta \mu_{1}^{*} \rho_{1}}{2 k^{2}} & -\frac{k}{s} \\
0 & 0 & 0 & C_{44} & -\frac{i \beta \rho_{1}\left(2 k^{2}+s^{2} \beta \rho_{1}\right)}{4 k^{3} d_{L}} & 0 \\
0 & -\frac{i \beta \rho_{2}\left(2 k^{2}+s^{2} \beta \rho_{2}\right)}{4 k^{3} d_{L}} & C_{53} & 0 & 0 & 0 \\
\frac{s \rho_{2}}{k} & \frac{s \mu_{2}^{*} \rho_{2}}{k^{2}} & C_{63} & C_{64} & \frac{s \mu_{1}^{*} \rho_{1}}{k^{2}} & \frac{s \rho_{1}}{k}
\end{array}\right]\left[\begin{array}{c}
\alpha_{2,1} \\
\alpha_{2,2} \\
\alpha_{2,3} \\
\alpha_{1,4} \\
\alpha_{1,5} \\
\alpha_{1,6}
\end{array}\right]=\left[\begin{array}{l}
0 \\
0 \\
0 \\
0 \\
0 \\
b
\end{array}\right],
$$

where

$$
\begin{aligned}
& C_{33}=\frac{\beta \varepsilon \rho_{2}\left(4\left(\varepsilon-i s d_{L}\right) k^{2}+3 s^{2} \beta \varepsilon \rho_{2}\right)-2 k^{4}\left(\varepsilon^{2}-2\right) d_{L}^{2}}{4 k^{3} \varepsilon^{2} d_{L}}, \\
& C_{34}=\frac{\beta \varepsilon \rho_{1}\left(4\left(\varepsilon+i s d_{L}\right) k^{2}+3 s^{2} \beta \varepsilon \rho_{1}\right)-2 k^{4}\left(\varepsilon^{2}-2\right) d_{L}^{2}}{4 k^{3} \varepsilon^{2} d_{L}}, \\
& C_{44}=\frac{i\left(4 d_{L}^{2} k^{4}+\beta \varepsilon \rho_{1}\left(2\left(\varepsilon+2 i s d_{L}\right) k^{2}+s^{2} \beta \varepsilon \rho_{1}\right)\right)}{4 k^{3} \varepsilon^{2} d_{L}}, \\
& C_{53}=\frac{i k d_{L}}{\varepsilon^{2}}+\frac{s \beta \rho_{2}}{k \varepsilon}+\frac{i \beta \rho_{2}\left(2 k^{2}+s^{2} \beta \rho_{2}\right)}{4 k^{3} d_{L}}, \\
& C_{63}=\frac{2 k d_{L}}{\beta \varepsilon^{2}}+\frac{\rho_{2}\left(4\left(\varepsilon-i s d_{L}\right) k^{2}+3 s^{2} \beta \varepsilon \rho_{2}\right)}{2 k^{3} \varepsilon d_{L}}, \\
& C_{64}=\frac{2 k d_{L}}{\beta \varepsilon^{2}}+\frac{\rho_{1}\left(4\left(\varepsilon+i s d_{L}\right) k^{2}+3 s^{2} \beta \varepsilon \rho_{1}\right)}{2 k^{3} \varepsilon d_{L}}
\end{aligned}
$$

and all entries are approximated up to $O\left(\varepsilon^{0}\right)$.

\section{APPENDIX C: ANALYTICAL LAPLACE TRANSFORM INVERSION}

In this section, we document the derivation of Eqs. (59) and (60). First, from Eqs. (35) and (56), the limiting interfacial growth is given by

$$
\frac{\eta(t)-\eta_{0}}{\mathcal{A} \eta_{0} k}=\mathcal{L}^{-1}\left[\frac{d_{L}}{s(1+\sqrt{1+\sigma(s)})} ; s \mapsto t\right],
$$

where $\sigma(s)=d_{L} s$ and the operator $\left(\mathcal{L}^{-1}: s \rightarrow t\right)$ is understood as the Bromwich integral given in Eq. (34). Using the cyclotron timescale, $T=t / d_{L}$, Eq. (C1) is equivalent to

$$
\frac{\hat{\eta}(T)-\hat{\eta}_{0}}{d_{L}}=\mathcal{L}^{-1}\left[\frac{1}{\sigma(1+\sqrt{1+\sigma})} ; \sigma \mapsto T\right],
$$

where $\hat{\eta}=\eta /\left(\mathcal{A} \eta_{0} k\right), \hat{\eta}_{0}=1 /(\mathcal{A} k)$, and the inversion operator now maps $\sigma$ in the Laplace space to $T$ in the time space. To proceed, we commit to the $\left(\mathcal{L}^{-1}: \sigma \rightarrow T\right)$ mapping and write

$$
\begin{aligned}
\frac{\hat{\eta}-\hat{\eta}_{0}}{d_{L}} & =\mathcal{L}^{-1}\left[\frac{1}{\sigma^{2}} \sqrt{1+\frac{1}{\sigma^{2}}}-\frac{1}{\sigma^{3}}\right] \\
& =\mathcal{L}^{-1}\left[\sum_{k=0}^{\infty}\left(\begin{array}{c}
1 / 2 \\
k
\end{array}\right) \sigma^{-2 k-2}-\frac{1}{\sigma^{3}}\right],
\end{aligned}
$$

where the binomial expansion is used. Now for each polynomial of $\sigma$, the Laplace inversion is standard, giving the desired result of Eq. (59), namely,

$$
\begin{aligned}
\frac{\hat{\eta}-\hat{\eta}_{0}}{d_{L}} & =\sum_{k=0}^{\infty}\left(\begin{array}{c}
1 / 2 \\
k
\end{array}\right) \frac{T^{2 k+1}}{(2 k+1) !}-\frac{T^{2}}{2} \\
& =T_{1} F_{2}\left(-\frac{1}{2} ; 1, \frac{3}{2} ;-\frac{T^{2}}{4}\right)-\frac{T^{2}}{2} .
\end{aligned}
$$

Similarly, the limiting interfacial circulation, $\hat{\gamma}_{0}(T)=\gamma_{0}(t) /$ $\left(4 \eta_{0} \mathcal{A}\right)$, follows from Eqs. (36) and (57) as:

$$
\begin{aligned}
\hat{\gamma}_{0}(T) & =\mathcal{L}^{-1}\left[\frac{\sigma \sqrt{\sigma^{2}+1}}{\left(\sqrt{\sigma^{2}+1}+1\right)\left(\cos ^{2} \phi+\sigma^{2}\right)}\right] \\
& =\mathcal{L}^{-1}\left[\frac{\sigma^{2}-\sigma+1}{\sigma \cos ^{2} \phi+\sigma^{3}}-\frac{\sqrt{\sigma^{2}+1}-\sigma}{\sigma \cos ^{2} \phi+\sigma^{3}}\right] .
\end{aligned}
$$


While the first term in (C5) can be inverted easily, inversion for the second term is achieved by the convolution of two standard integrals

$$
\begin{aligned}
\mathcal{L}\left[\frac{J_{1}(T)}{T}\right] & =\sqrt{\sigma^{2}+1}-\sigma, \\
\mathcal{L}\left[\frac{1-\cos (T \cos \phi)}{\cos ^{2} \phi}\right] & =\frac{1}{\sigma \cos ^{2} \phi+\sigma^{3}} .
\end{aligned}
$$

As a result, Eq. (60) in the closed form follows:

$$
\begin{aligned}
\hat{\gamma}_{0}= & \frac{\left(\cos ^{2} \phi-1\right) \cos (T \cos \phi)-\cos \phi \sin (T \cos \phi)+1}{\cos ^{2} \phi} \\
& -\frac{1-\cos (T \cos \phi)}{\cos ^{2} \phi} * \frac{J_{1}(T)}{T} .
\end{aligned}
$$

\section{APPENDIX D: LINEARIZED RANKINE-HUGONIOT CONDITIONS}

For the purpose of this discussion, we consider a real valued interfacial perturbation profile, $h(x, t)=\eta(t) \cos (k x)$, where $1 \gg \eta(t) \in \mathbb{R}$. The corresponding unit tangent and normal vectors along the interface in the $x, z$-plane are thus

$$
\hat{\boldsymbol{t}}=\frac{1}{1+\eta^{2} k^{2} \sin ^{2}(k x)}(1,0,-\eta k \sin (k x))=(1,0,-K \eta)+O\left(\eta^{2}\right),
$$

$$
\hat{\boldsymbol{n}}=\frac{1}{1+\eta^{2} k^{2} \sin ^{2}(k x)}(\eta k \sin (k x), 0,-1)=(K \eta, 0,1)+O\left(\eta^{2}\right),
$$

where $K=k \sin (k x)$ is a constant.

The background magnetic field can be expressed as

$$
\boldsymbol{B}_{0}=\left(\sqrt{1-\epsilon^{2}}, 0, \epsilon\right), \quad 0 \leq \epsilon \leq 1 .
$$

However in the following, we specialize to the case where $\epsilon \ll 1$. For a sufficiently small $\eta$, the perturbed magnetic field that results from the initial density interface distortion of magnitude $\eta_{0}[=O(\eta)]$ can be expanded in a perturbation series

$$
\begin{aligned}
\boldsymbol{B} & =\boldsymbol{B}_{0}+\boldsymbol{B}_{1} \eta_{0}+O\left(\eta_{0}^{2}\right) \\
& =\left(1+O\left(\epsilon^{2}\right)+B_{1 x} \eta_{0}, B_{1 y} \eta_{0}, \epsilon+B_{1 z} \eta_{0}\right)+O\left(\eta_{0}^{2}\right),
\end{aligned}
$$

where $\boldsymbol{B}_{1}=\boldsymbol{B}_{1}(x, z, t)$ is of the order unity. Extracting normal and tangential components of the field to the first order gives

$$
\begin{gathered}
B_{n}=\boldsymbol{B} \cdot \hat{\boldsymbol{n}}=K \eta+\epsilon+B_{1 z} \eta_{0}, \\
B_{t}=\boldsymbol{B} \cdot \hat{\boldsymbol{t}}=1+B_{1 x} \eta_{0} .
\end{gathered}
$$

As a reminder, the nonlinear Rankine-Hugoniot CD jump conditions listed in (4) include

$$
\begin{gathered}
\llbracket\left(p+\frac{1}{\beta} B^{2}\right) \hat{\boldsymbol{n}}-\frac{2}{\beta} B_{n} \boldsymbol{B} \rrbracket=0, \\
\llbracket B_{n} \rrbracket=0
\end{gathered}
$$

which apply to both regular MHD and Hall-MHD systems. In particular, using (D5) and (D6), the tangential component of (D7) requires

$$
\llbracket B_{n} B_{t} \rrbracket=\left[B_{1 z} \eta_{0}+B_{1 x} \eta_{0} \epsilon+O\left(\eta_{0}^{2}\right)\right]=0 .
$$

To be consistent with the linearization of the governing equations discussed in Sec. II C, the CD jump conditions must be imposed at the order $O\left(\eta_{0}\right)$. It follows that the first term in (D9) automatically drops due to (D8), and (D9) is non-trivial only if $\eta_{0} \ll \epsilon$, in which case, continuity of $B_{1 x}$ is strictly required. Otherwise, (D9) is equivalent to (D8) to the leading order, and even though the unperturbed magnetic field lines still cut through the $\mathrm{CD}$, continuity of $B_{1 x}$ should not be demanded in the linear region.

This analysis therefore justifies the use of correct boundary conditions given in Eq. (17) where $\epsilon=0$, and (32) where $\eta_{0} \ll \epsilon \ll 1$.

\section{DATA AVAILABILITY}

The data that support the findings of this study are available from the corresponding author upon reasonable request.

\section{REFERENCES}

${ }^{1}$ R. D. Richtmyer, "Taylor instability in shock acceleration of compressible fluids," Commun. Pure Appl. Math. 13, 297-319 (1960).

${ }^{2}$ E. E. Meshkov, "Instability of the interface of two gases accelerated by a shock wave," Fluid Dyn. 4, 101-104 (1972).

${ }^{3} \mathrm{D}$. Arnett, "The role of mixing in astrophysics," Astrophys. J. Suppl. Ser. 127, 213 (2000).

${ }^{4}$ A. M. Khokhlov, E. S. Oran, and G. O. Thomas, "Numerical simulation of deflagration-to-detonation transition: The role of shock-flame interactions in turbulent flames," Combust. Flame 117, 323-339 (1999).

${ }^{5}$ J. Yang, T. Kubota, and E. E. Zukoski, "Applications of shock-induced mixing to supersonic combustion,” AIAA J. 31, 854-862 (1993).

${ }^{6}$ M. Brouillette, “The Richtmyer-Meshkov instability," Annu. Rev. Fluid Mech. 34, 445-468 (2002).

${ }^{7}$ S. H. Glenzer, B. J. MacGowan, P. Michel, N. B. Meezan, L. J. Suter, S. N. Dixit, J. L. Kline, G. A. Kyrala, D. K. Bradley, D. A. Callahan, E. L. Dewald, L. Divol, E. Dzenitis, M. J. Edwards, A. V. Hamza, C. A. Haynam, D. E. Hinkel, D. H. Kalantar, J. D. Kilkenny, O. L. Landen, J. D. Lindl, S. LePape, J. D. Moody, A. Nikroo, T. Parham, M. B. Schneider, R. P. J. Town, P. Wegner, K. Widmann, P. Whitman, B. K. F. Young, B. Van Wonterghem, L. J. Atherton, and E. I. Moses, "Symmetric inertial confinement fusion implosions at ultra-high laser energies," Science 327, 1228 (2010).

${ }^{8}$ J. D. Lindl, R. L. McCrory, and E. M. Campbell, "Progress toward ignition and burn propagation in inertial confinement fusion," Phys. Today 45(9), 32-40 (1992).

${ }^{9}$ N. E. Lanier, C. W. Barnes, S. H. Batha, R. D. Day, G. R. Magelssen, J. M. Scott, A. M. Dunne, K. W. Parker, and S. D. Rothman, "Multimode seeded Richtmyer-Meshkov mixing in a convergent, compressible, miscible plasma system,” Phys. Plasmas 10, 1816 (2003).

${ }^{10}$ S. H. R. Hosseini and K. Takayama, "Experimental study of RichtmyerMeshkov instability induced by cylindrical shock waves," Phys. Fluids 17, 084101 (2005).

${ }^{11}$ K. O. Mikaelian, "Rayleigh-Taylor and Richtmyer-Meshkov instabilities and mixing in stratified cylindrical shells," Phys. Fluids 17, 094105 (2005).

${ }^{12}$ M. Lombardini, D. I. Pullin, and D. I. Meiron, "Turbulent mixing driven by spherical implosions. Part 1. Flow description and mixing-layer growth," J. Fluid Mech. 748, 85-112 (2014).

${ }^{13}$ M. Lombardini, D. I. Pullin, and D. I. Meiron, "Turbulent mixing driven by spherical implosions. Part 2. Turbulence statistics,” J. Fluid Mech. 748, 113-142 (2014).

${ }^{14} \mathrm{~A}$. Bakhsh and R. Samtaney, "Incompressible models of magnetohydrodynamic Richtmyer-Meshkov instability in cylindrical geometry," Phys. Rev. Fluids 4, 063906 (2019). 
${ }^{15}$ A. López Ortega, M. Lombardini, D. I. Pullin, and D. I. Meiron, "Numerical simulations of the Richtmyer-Meshkov instability in solid-vacuum interfaces using calibrated plasticity laws," Phys. Rev. E 89, 033018 (2014).

${ }^{16}$ A. López Ortega, M. Lombardini, P. T. Barton, D. I. Pullin, and D. I. Meiron, "Richtmyer-Meshkov instability for elastic-plastic solids in converging geometries," J. Mech. Phys. Solids 76, 291-324 (2015).

${ }^{17}$ M.-E. Manuel, C. K. Li, F. H. Séguin, J. Frenje, D. T. Casey, R. D. Petrasso, S. X. Hu, R. Betti, J. D. Hager, D. D. Meyerhofer et al., "First measurements of rayleigh-taylor-induced magnetic fields in laser-produced plasmas," Phys. Rev. Lett. 108, 255006 (2012).

${ }^{18}$ F. H. Séguin, C. K. Li, M.-E. Manuel, H. G. Rinderknecht, N. Sinenian, J. A. Frenje, J. R. Rygg, D. G. Hicks, R. D. Petrasso, J. Delettrez et al., "Time evolution of filamentation and self-generated fields in the coronae of directly driven inertial-confinement fusion capsules," Phys. Plasmas 19, 012701 (2012).

${ }^{19}$ I. V. Igumenshchev, A. B. Zylstra, C. K. Li, P. M. Nilson, V. N. Goncharov, and R. D. Petrasso, "Self-generated magnetic fields in direct-drive implosion experiments," Phys. Plasmas 21, 062707 (2014).

${ }^{20}$ D. Bond, V. Wheatley, R. Samtaney, and D. I. Pullin, "Richtmyer-Meshkov instability of a thermal interface in a two-fluid plasma," J. Fluid Mech. 833, 332-363 (2017).

${ }^{21}$ M. Hohenberger, P.-Y. Chang, G. Fiskel, J. P. Knauer, R. Betti, F. J. Marshall, D. D. Meyerhofer, F. H. Séguin, and R. D. Petrasso, "Inertial confinement fusion implosions with imposed magnetic field compression using the OMEGA laser," Phys. Plasmas 19, 056306 (2012).

${ }^{22}$ L. J. Perkins, B. G. Logan, G. B. Zimmerman, and C. J. Werner, "Two-dimensional simulations of thermonuclear burn in ignition-scale inertial confinement fusion targets under compressed axial magnetic fields," Phys. Plasmas 20, 072708 (2013).

${ }^{23}$ R. Samtaney, "Suppression of the Richtmyer-Meshkov instability in the presence of a magnetic field," Phys. Fluids 15, L53-L56 (2003).

${ }^{24} \mathrm{~V}$. Wheatley, D. I. Pullin, and R. Samtaney, "Stability of an impulsively accelerated density interface in magnetohydrodynamics," Phys. Rev. Lett. 95, 125002 (2005).

${ }^{25} \mathrm{~V}$. Wheatley, R. Samtaney, and D. I. Pullin, “The Richtmyer-Meshkov instability in magnetohydrodynamics," Phys. Fluids 21, 082102-082113 (2009).

${ }^{26} \mathrm{~J}$. Cao, Z. Wu, H. Ren, and D. Li, "Effects of shear flow and transverse magnetic field on Richtmyer-Meshkov instability," Phys. Plasmas 15, 042102 (2008).
${ }^{27}$ V. Wheatley, R. Samtaney, D. I. Pullin, and R. M. Gehre, “The transverse field Richtmyer-Meshkov instability in magnetohydrodynamics," Phys. Fluids 26, 016102-016117 (2014).

${ }^{28}$ W. Mostert, V. Wheatley, R. Samtaney, and D. I. Pullin, "Effects of seed magnetic fields on magnetohydrodynamic implosion structure and dynamics," Phys. Fluids 26, 126102 (2014).

${ }^{29}$ W. Mostert, V. Wheatley, R. Samtaney, and D. I. Pullin, "Effects of magnetic fields on magnetohydrodynamic cylindrical and spherical Richtmyer-Meshkov instability," Phys. Fluids 27, 104102 (2015).

${ }^{30} \mathrm{~N}$. Shen, D. I. Pullin, V. Wheatley, and R. Samtaney, "Impulse-driven Richtmyer-Meshkov instability in Hall-magnetohydrodynamics," Phys. Rev. Fluids 4, 103902 (2019).

${ }^{31}$ N. Shen, Y. Li, D. I. Pullin, R. Samtaney, and V. Wheatley, "On the magnetohydrodynamic limits of the ideal two-fluid plasma equations," Phys. Plasmas 25, 122113 (2018).

${ }^{32}$ J. D. Huba, "Theory and simulation of a high-frequency magnetic drift wave," Phys. Fluids B: Plasma Phys. 3, 3217-3225 (1991).

${ }^{33}$ J. D. Huba, "Hall magnetohydrodynamics-a tutorial," Space Plasma Simulation (Springer, 2003), pp. 166-192.

${ }^{34}$ E. Hameiri, A. Ishizawa, and A. Ishida, "Waves in the Hallmagnetohydrodynamics model,” Phys. Plasmas 12, 072109 (2005).

${ }^{35}$ V. Wheatley, R. M. Gehre, R. Samtaney, and D. I. Pullin, The Magnetohydrodynamic Richtmyer-Meshkov Instability: The Oblique Field Case (Springer, 2013), pp. 1107-1112.

${ }^{36}$ M. Abramowitz and I. A. Stegun, Handbook of Mathematical Functions: With Formulas, Graphs, and Mathematical Tables (Courier Corporation, 1965), Vol. 55.

${ }^{37}$ D. P. Gaver, Jr., "Observing stochastic processes, and approximate transform inversion,” Oper. Res. 14, 444-459 (1966).

${ }^{38}$ H. Stehfest, "Algorithm 368: Numerical inversion of Laplace transforms, [d5]," Commun. ACM 13, 47-49 (1970).

${ }^{39} \mathrm{~J}$. Abate and W. Whitt, "A unified framework for numerically inverting Laplace transforms," Informs J. Comput. 18, 408-421 (2006).

${ }^{40}$ R. Samtaney, P. Colella, T. J. Ligocki, D. F. Martin, and S. C. Jardin, "An adaptive mesh semi-implicit conservative unsplit method for resistive MHD," J. Phys.: Conf. Ser. 16, 40 (2005). 